May 10, 2016 0:33 WSPC/INSTRUCTION FILE DQPM-review

International Journal of Modern Physics E

(C) World Scientific Publishing Company

\title{
A dynamical quasiparticle approach for the Quark-Gluon-Plasma bulk and transport properties
}

\author{
Hamza Berrehrah* \\ Frankfurt Institute for Advanced Studies, Johann Wolfgang Goethe Universität, \\ Ruth-Moufang-Strasse 1, 60438 Frankfurt am Main, Germany \\ Institute for Theoretical Physics, Johann Wolfgang Goethe Universität, Max-von-Laue-Str. 1, \\ 60438 Frankfurt am Main, Germany \\ Elena Bratkovskaya ${ }^{\dagger}$ \\ Frankfurt Institute for Advanced Studies, Johann Wolfgang Goethe Universität, \\ Ruth-Moufang-Strasse 1, 60438 Frankfurt am Main, Germany \\ Institute for Theoretical Physics, Johann Wolfgang Goethe Universität, Max-von-Laue-Str. 1, \\ 60438 Frankfurt am Main, Germany \\ GSI Helmholtzzentrum für Schwerionenforschung GmbH, 64291 Darmstadt, Germany \\ Thorsten Steinert $\ddagger$ Wolfgang Cassing ${ }^{\S}$ \\ Institut für Theoretische Physik, Universität Gießen, Heinrich-Buff-Ring 16, 35392 Gießen, \\ Germany
}

Received Day Month Year Revised Day Month Year

\begin{abstract}
The properties of quantum-chromo dynamics (QCD) nowadays are accessable by lattice QCD calculations at vanishing quark chemical potential $\mu_{q}=0$ but often lack a transparent physical interpretation. In this review we report about results from an extended dynamical quasiparticle model (DQPM*) in which the effective parton propagators have a complex selfenergy that depends on the temperature $T$ of the medium as well as on the chemical potential $\mu_{q}$ and the parton three-momentum $\boldsymbol{p}$ with respect to the medium at rest. It is demonstrated that this approach allows for a good description of QCD thermodynamics with respect to the entropy density, pressure etc. above the critical temperature $T_{c} \approx 158 \mathrm{MeV}$. Furthermore, the quark susceptibility $\chi_{q}$ and the quark number density $n_{q}$ are found to be reproduced simultaneously at zero and finite quark chemical potential. The shear and bulk viscosities $\eta, \zeta$, and the electric conductivity $\sigma_{e}$ from the DQPM* also turn out in close agreement with lattice results for $\mu_{q}=0$. The $\mathrm{DQPM}^{*}$, furthermore, allows to evaluate the momentum $p, T$ and $\mu_{q}$ dependencies of the partonic degrees of freedom also for larger $\mu_{q}$ which are mandatory for transport studies of heavy-ion collisions in the regime $5 \mathrm{GeV}<\sqrt{s_{N N}}<10 \mathrm{GeV}$. We finally calculate the charm quark diffusion coefficient $D_{s}$ - evaluated from the differential cross sections
\end{abstract}

*berrehrah@fias.uni-frankfurt.de

$\dagger$ E.Bratkovskaya@gsi.de

¥thorsten.steinert@theo.physik.uni-giessen.de

$\S_{\text {wolfgang.cassing@theo.physik.uni-giessen.de }}$ 
of partons in the medium for light and heavy quarks by employing the propagators and couplings from the DQPM - and compare to the available lattice data. It is argued that the complete set of observables allows for a transparent interpretation of the properties of hot QCD.

Keywords: Quark Gluon Plasma, Susceptibility, Cross sections, Collisional processes, pQCD, QCD, On-shell, Off-shell.

PACS numbers:24.10.Jv, 02.70.Ns, 12.38.Mh, 24.85.+p

\section{Contents}

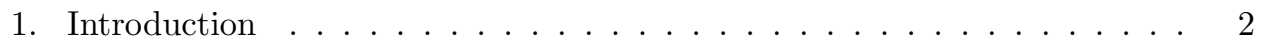

2. Parton properties in the $\mathrm{DQPM}^{*} \ldots \ldots \ldots \ldots$ 4 . . . . . .

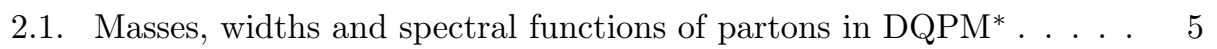

2.2. The running coupling in $\mathrm{DQPM}^{*} \ldots \ldots \ldots \ldots$. . . . . . 7

2.3. Thermodynamics of the QGP from $\mathrm{DQPM}^{*} \ldots \ldots \ldots \ldots$

3. Quark number density and susceptibility from DQPM* . . . . . . . . 11

3.1. Baryon number density in the DQPM $\ldots \ldots \ldots \ldots$. . . . . . 11

3.2. Susceptibilities in the $\mathrm{DQPM}^{*} \ldots \ldots \ldots \ldots \ldots \ldots$

3.3. $n_{B}$ and $\chi_{q}$ : DQPM $^{*}$ vs lQCD . . . . . . . . . . . 13

4. Transport properties of the hot QGP from DQPM $\ldots \ldots \ldots \ldots$

4.1. Shear and bulk viscosities . . . . . . . . . . . . . . . 13

4.2. Electric conductivity . . . . . . . . . . . . . 17

5. Differential partonic cross sections and transport coefficients for charm

quarks in the hot medium . . . . . . . . . . . . . . . 18

5.1. $q Q$ and $g Q$ elastic scattering at finite $T$ and $\mu_{q} \ldots \ldots \ldots$

5.2. Heavy quark interaction rates in a medium at finite $T$ and $\mu_{q} \ldots 21$

5.3. Diffusion coefficient and energy loss of charm quarks in the hot medium 23

6 . Summary . . . . . . . . . . . . . . . . . . 26 26

\section{Introduction}

The thermodynamic properties of the quark-gluon plasma (QGP)-as produced in relativistic heavy-ion collisions-are rather well determined within lattice QCD (lQCD) calculations at vanishing quark chemical potential $\mu_{q}{ }^{[1] 6]}$ Whereas the results from different collaborations in the past have led to different equations of state (EoS) of partonic matter even at $\mu_{q}=0$ the present status can be considered as a consensus (within error bars). Nevertheless, the physical interpretation of the lattice 'data' remains a challenge since the EoS as well as transport coefficients from IQCD indicate that the partonic system cannot be viewed as a weakly interacting medium of quark, antiquarks, and gluons. This holds especially true for temperatures close to the critical temperature $T_{c}$ where the entropy density $s(T)$ (and pressure $P(T)$ ) differ substantially from the Stefan Boltzmann limit. The lQCD results on the EoS can conveniently be interpreted within quasiparticle models with massive partons ${ }^{7 / 14}$ that are fitted to the equation of state (EoS) from IQCD and 
also allow for extrapolations to finite $\mu_{q}$, although with some ambiguities. However, in these effective models the spectral function of the degrees of freedom is taken as a $\delta$ - function (on-shell limit) which implies that these partons in principle are non-interacting. An extension of the simple quasiparticle model has been proposed in Refs. $\frac{15}{18}$ where a finite width of the partonic spectral functions is introduced, which corresponds to the interaction rate of the parton in the medium at finite temperature $T$ and chemical potential $\mu_{q}$. The latter can be directly employed for the calculation of transport coefficients such as shear and bulk viscosities of the partonic medium in the relaxation time approximation ${ }^{19}$ and be compared to corresponding correlators from lQCD. An interpretation in terms of quasiparticles, however, is constraint to effective propagators with a spectral width that is substantially smaller than the dynamical pole mass.

Furthermore, at non-zero quark chemical potential $\mu_{q} \neq 0$, the primary quantities of interest are the "pressure difference $\Delta P$ ", the quark number density $n_{B}$ and quark susceptibility $\chi_{q}$ since these quantities are available from 1QCD 20$] 21$ The quark number susceptibilities are additional quantities to further quantify the properties of the partonic degrees of freedom ( d.o.f.) especially in the vicinity of the QCD phase transition or crossover $4 \sqrt[4]{22}$ It turns out that the standard quasiparticle models, that fit the partonic EoS, severely underestimate the quark susceptibilities. Nevertheless, the challenge of describing simultaneously both the lQCD pressure and quark susceptibilities as well as transport coefficients is out of reach in these model ${ }^{13}$ which has been pointed out in particular in Refs ${ }^{13}[14$ Especially the quark susceptibilities are very sensitive to the quark masses used as inputs and solely determined by the quark degrees of freedom. On the other hand both light quark and gluon masses contribute to thermodynamic quantities like the entropy density $s$ and pressure $P$. Therefore, reconciling all observables from lQCD within a single effective model is a challenge.

Apart from the interactions in the light quark sector - dominating the partonic equation of state - also the properties of heavy charm quarks are of interest since their drag and diffusion controls the elliptic flow $v_{2}$ of charm quarks as well as the suppression at high transverse momentum ${ }^{23} \sqrt{24}$ in relativistic heavy-ion reactions. Although the charm quarks can be considered as reasonable quasiparticles - with a pole mass that is large compared to the spectral width - the interactions with the nonperturbative bulk partons are of interest and in particular the transport coefficient $\hat{q}$ and the $c$-quark drag coefficient as a function of $T$ and $\mu_{q}$.

In this review we will consider the QGP as a dynamical quasi-particle medium of massive off-shell particles with partonic propagators incorporating complex selfenergies which explicitly depend on the three-momentum $\boldsymbol{p}$ with respect to the partonic matter at rest in order to match perturbative QCD (pQCD) at high momenta. We will show that within the extended dynamical quasiparticle model denoted by $\mathrm{DQPM}^{*}$ - we reproduce the lQCD equation of state at finite temperature $T$ and chemical potential $\mu_{q}$. Moreover, we simultaneously describe the quark number density and susceptibility $\chi_{q}$ from lQCD. In the same approach, we also 
compute the shear and bulk viscosities $(\eta$ and $\zeta$ ), and the electric conductivity $\left(\sigma_{e}\right)$ of the QGP at finite temperature $T$ and chemical potential $\mu_{q}$ in order to probe some transport properties of the partonic medium in analogy to the studies in Refs. 25 The partonic spectral functions (or imaginary parts of the retarded propagators) at finite temperature and chemical potential are determined for these dynamical quasi-particles and the shear viscosity $\eta$ and bulk viscosity $\zeta$ is computed within the relaxation-time approximation (RTA) which provides similar results as the Green-Kubo method employed in Refs $15,29,30$

The review is organized as follows: We first present in Section 2 the basic ingredients of the QGP d.o.f in terms of their masses and widths, which are the essential ingredients in their retarded propagators, as well as the running coupling (squared) $g^{2}\left(T, \mu_{q}\right)$. The gluon and fermion propagators - as given by the DQPM* at finite three-momentum $\boldsymbol{p}$, temperature $T$ and quark chemical potential $\mu_{q}-$ contain a few parameters that are fixed in comparison to results from lQCD. As a first application we will compute the 1QCD pressure and interaction measure in a partonic medium at finite $T$ and $\mu_{q}$ and compare to related results from lattice QCD. In Section 3 we investigate the quark number density and susceptibility within the DQPM* and compare to lQCD results for $2+1$ flavors $\left(N_{f}=3\right)$. In Section 4 we compute the QGP shear and bulk viscosities as well as the electric conductivity and compare to IQCD results and other theoretical studies. Throughout Sections 2-4 we will point out the importance of finite masses and widths of the light dynamical quasiparticles, including their finite momentum, temperature and $\mu_{q}$ dependencies. Section 5 is devoted to the dynamics and transport properties of heavy charm quarks in the hot and dense medium. To this aim we calculate the differential cross sections between the light and heavy partons - on the basis of the standard DQPM couplings and propagators - and evaluate their interaction rates in the quasiparticle limit. Furthermore, we compute the spatial diffusion coefficient and energy loss of the charm degrees of freedom and compare to the available lQCD data. In Section 6 , finally, we summarize the main results and point out the future applications of the DQPM*.

\section{Parton properties in the DQPM*}

In the DQPM* the entropy density $s(T)$, the pressure $P(T)$ and energy density $\epsilon(T)$ are calculated in a straight forward manner by starting with the entropy density in the quasiparticle limit from Baym, $18|31| 32$

$$
\begin{aligned}
& s^{d q p}=-d_{g} \int \frac{d \omega}{2 \pi} \frac{d^{3} p}{(2 \pi)^{3}} \frac{\partial f_{B}}{\partial T}\left(\Im \ln \left(-\Delta^{-1}\right)+\Im \Pi \Re \Delta\right) \\
& -d_{q} \int \frac{d \omega}{2 \pi} \frac{d^{3} p}{(2 \pi)^{3}} \frac{\partial f_{F}\left(\left(\omega-\mu_{q}\right) / T\right)}{\partial T}\left(\Im \ln \left(-S_{q}^{-1}\right)+\Im \Sigma_{q} \Re S_{q}\right) \\
& -d_{\bar{q}} \int \frac{d \omega}{2 \pi} \frac{d^{3} p}{(2 \pi)^{3}} \frac{\partial f_{F}\left(\left(\omega+\mu_{q}\right) / T\right)}{\partial T}\left(\Im \ln \left(-S_{\bar{q}}^{-1}\right)+\Im \Sigma_{\bar{q}} \Re S_{\bar{q}}\right),
\end{aligned}
$$


where $f_{B}(\omega / T)=(\exp (\omega / T)-1)^{-1}$ and $f_{F}\left(\left(\omega-\mu_{q}\right) / T\right)=\left(\exp \left(\left(\omega-\mu_{q}\right) / T\right)+\right.$ $1)^{-1}$ denote the Bose and Fermi distribution functions, respectively, while $\Delta=$ $\left(P^{2}-\Pi\right)^{-1}, S_{q}=\left(P^{2}-\Sigma_{q}\right)^{-1}$ and $S_{\bar{q}}=\left(P^{2}-\Sigma_{\bar{q}}\right)^{-1}$ stand for the full (scalar) quasiparticle propagators of gluons $g$, quarks $q$ and antiquarks $\bar{q}$. In Eq. (1) $\Pi$ and $\Sigma=\Sigma_{q} \approx \Sigma_{\bar{q}}$ denote the (retarded) quasiparticle selfenergies. In principle, $\Pi$ as well as $\Delta$ are Lorentz tensors and should be evaluated in a nonperturbative framework. The DQPM* treats these degrees of freedom as independent scalar fields with scalar selfenergies which are assumed to be identical for quarks and antiquarks. Note that one has to treat quarks and antiquarks separately in Eq. (1) as their abundance differs at finite quark chemical potential $\mu_{q}$. In Eq. (1) the degeneracy for gluons is $d_{g}=2\left(N_{c}^{2}-1\right)=16$ while $d_{q}=d_{\bar{q}}=2 N_{c} N_{f}=18$ is the degeneracy for quarks and antiquarks with three flavors. In practice one also has to differentiate between $(u, d)$ and $s$ quarks due to their mass difference.

As a next step one writes the complex selfenergies as $\Pi(\boldsymbol{q})=M_{g}^{2}(\boldsymbol{q})-2 i \omega \gamma_{g}(\boldsymbol{q})$ and $\Sigma_{q}(\boldsymbol{q})=M_{q}(\boldsymbol{q})^{2}-2 i \omega \gamma_{q}(\boldsymbol{q})$ with a mass (squared) term $M^{2}$ and an interaction width $\gamma$, i.e. the retarded propagators $\left(\Delta, S_{q}\right)$ read,

$$
G_{R}(\omega, \boldsymbol{q})=\left(\omega^{2}-\boldsymbol{q}^{2}-M^{2}(\boldsymbol{q})+2 i \gamma(\boldsymbol{q}) \omega\right)^{-1},
$$

and are analytic in the upper half plane in the energy $\omega$ since the poles of $G_{R}$ are located in the lower half plane. The imaginary part of $G_{R}(2)$ then gives the spectral function of the degree of freedom (except for a factor $1 / \pi$ ). In the standard DQPM $18 \sqrt[33]{34}$ the masses had been fixed in the spirit of the hard thermal loop (HTL) approach with the masses being proportional to an effective coupling $g\left(T / T_{c}\right)$ which has been enhanced in the infrared. In the DQPM* the selfenergies depend additionally on the three-momentum $\boldsymbol{p}$ with respect to the medium at rest, while the dependence on the temperature $T / T_{c}$ and chemical potential $\mu_{q}$ are very similar to the standard DQPM 18 [3] 34

\subsection{Masses, widths and spectral functions of partons in $D Q P M^{*}$}

The functional forms for the parton masses and widths at finite temperature $T$, quark chemical potential $\mu_{q}$ and momentum $p=|\boldsymbol{p}|$ are assumed to be given by

$$
\begin{aligned}
& M_{g}\left(T, \mu_{q}, p\right)=\left(\frac{3}{2}\right)\left[\frac{g^{2}\left(T^{\star} / T_{c}\left(\mu_{q}\right)\right)}{6}\left[\left(N_{c}+\frac{N_{f}}{2}\right) T^{2}+\frac{N_{c}}{2} \sum_{q} \frac{\mu_{q}^{2}}{\pi^{2}}\right]\right]^{1 / 2} \times h\left(\Lambda_{g}, p\right)+m_{\chi g}, \\
& M_{q, \bar{q}}\left(T, \mu_{q}, p\right)=\left[\frac{N_{c}^{2}-1}{8 N_{c}} g^{2}\left(T^{\star} / T_{c}\left(\mu_{q}\right)\right)\left[T^{2}+\frac{\mu_{q}^{2}}{\pi^{2}}\right]\right]^{1 / 2} \times h\left(\Lambda_{q}, p\right)+m_{\chi q}, \\
& \gamma_{g}\left(T, \mu_{q}, p\right)=N_{c} \frac{g^{2}\left(T^{\star} / T_{c}\left(\mu_{q}\right)\right)}{8 \pi} T \ln \left(\frac{2 c}{g^{2}\left(T^{\star} / T_{c}\left(\mu_{q}\right)\right)}+1.1\right)^{3 / 4} \times h\left(\Lambda_{g}, p\right), \\
& \gamma_{q, \bar{q}}\left(T, \mu_{q}, p\right)=\frac{N_{c}^{2}-1}{2 N_{c}} \frac{g^{2}\left(T^{\star} / T_{c}\left(\mu_{q}\right)\right)}{8 \pi} T \ln \left(\frac{2 c}{g^{2}\left(T^{\star} / T_{c}\left(\mu_{q}\right)\right)}+1.1\right)^{3 / 4} \times h\left(\Lambda_{q}, p\right),
\end{aligned}
$$


with the momentum-dependent function

$$
h(\Lambda, p)=\left[\frac{1}{1+\Lambda\left(T_{c}\left(\mu_{q}\right) / T^{\star}\right) p^{2}}\right]^{1 / 2},
$$

where $T^{\star 2}=T^{2}+\mu_{q}^{2} / \pi^{2}$ is the effective temperature used to extend the DQPM* to finite $\mu_{q}$, while $\Lambda_{g}\left(T_{c}\left(\mu_{q}\right) / T^{\star}\right)=5\left(T_{c}\left(\mu_{q}\right) / T^{\star}\right)^{2} \mathrm{GeV}^{-2}$ and $\Lambda_{q}\left(T_{c}\left(\mu_{q}\right) / T^{\star}\right)=$ $12\left(T_{c}\left(\mu_{q}\right) / T^{\star}\right)^{2} \mathrm{GeV}^{-2}$. Furthermore, $m_{\chi g} \approx 0.5 \mathrm{GeV}$ is the gluon condensate and $m_{\chi q}$ is the light-quark chiral mass $\left(m_{\chi q}=0.003 \mathrm{GeV}\right.$ for $u, d$ quarks and $m_{\chi q}=0.06$ $\mathrm{GeV}$ for $s$ quarks). Since the effective quark masses in the QGP are large compared to the chiral masses the latter can in practice be neglected. In Eq. (3) $m_{\chi g}\left(m_{\chi q}\right)$ gives the finite gluon (light quark) mass in the limit $p \rightarrow 0$ and $T=0$ or for $p \rightarrow$ $\infty$. As mentioned above the quasiparticle masses and widths (3) are parametrized following hard thermal loop (HTL) functional dependencies at finite temperature as in the default DQPM ${ }^{18}$ in order to follow the correct high temperature limit. The essentially new elements in (3) are the multiplicative factors $h(\Lambda, p)$ (4) specifying the momentum dependence of the masses and widths with additional parameters $\Lambda_{g}$ and $\Lambda_{q}$ and the additive terms $m_{\chi g}$ and $m_{\chi q}$. The momentum-dependent factor $h(\Lambda, p)$ in the masses $(3)$ is motivated by Dyson-Schwinger studies in the vacuum $\frac{35}{35}$ and yields the limit of pQCD for $p \rightarrow \infty$.

The effective gluon and quark masses are a function of $T^{\star}$ at finite $\mu_{q}$. Here we consider three light flavors $(q=u, d, s)$ and assume all chemical potentials to be equal $\left(\mu_{u}=\mu_{d}=\mu_{s}=\mu_{q}\right)$. Note that alternative settings are also possible to comply with strangeness neutrality in heavy-ion collisions. The coupling (squared) $g^{2}$ in Eq. (3) is the effective running coupling given as a function of $T / T_{c}$ at $\mu_{q}=0$. A straight forward extension of the DQPM ${ }^{*}$ to finite $\mu_{q}$ is to consider the coupling as a function of $T^{\star} / T_{c}\left(\mu_{q}\right)$ with a $\mu_{q}$-dependent critical temperature $T_{c}\left(\mu_{q}\right)$,

$$
T_{c}\left(\mu_{q}\right)=T_{c}\left(\mu_{q}=0\right) \sqrt{1-\alpha \mu_{q}^{2}} \approx T_{c}\left(\mu_{q}=0\right)\left(1-\alpha / 2 \mu_{q}^{2}+\ldots\right)
$$

with $\alpha \approx 8.79 \mathrm{GeV}^{-2}$. We recall that the expression of $T_{c}\left(\mu_{q}\right)$ in Eq. (5) is obtained by requiring a constant energy density $\epsilon$ for the system at $T=T_{c}\left(\mu_{q}\right)$ where $\epsilon$ at $T_{c}\left(\mu_{q}=0\right) \approx 0.158 \mathrm{GeV}$ is fixed by a lattice QCD calculation at $\mu_{q}=0$. The coefficient in front of the $\mu_{q}^{2}$-dependent part can be compared to lQCD calculations at finite (but small) $\mu_{B}$ which gives 36

$$
T_{c}\left(\mu_{B}\right)=T_{c}\left(\mu_{B}=0\right)\left(1-\kappa\left(\frac{\mu_{B}}{T_{c}\left(\mu_{B}=0\right)}\right)^{2}+\ldots\right)
$$

with $\kappa=0.013(2)$. Rewriting (5) in the form (6) and using $\mu_{B} \approx 3 \mu_{q}$ we get $\kappa_{D Q P M} \approx 0.0122$ which compares well with the lQCD result.

Using the pole masses and widths (3), the spectral functions for the partonic degrees of freedom are fully determined, i.e. the imaginary parts of the retarded propagators. The real part of the retarded propagators then follows from dispersion relations or directly from Eq. (2). Since the retarded propagators show no poles in 
the upper complex half plane in the energy $\omega$ the model propagators obey microcausality $\stackrel{[37}{[1}$ The imaginary parts are of Lorentzian form and provide the spectral functions $16\left[\begin{array}{l|l|l|l|}16 & 38 \\ \hline\end{array}\right.$

$$
\rho_{i}(\omega, \boldsymbol{p})=\frac{\gamma_{i}(\boldsymbol{p})}{\tilde{E}_{i}(\boldsymbol{p})}\left(\frac{1}{\left(\omega-\tilde{E}_{i}(\boldsymbol{p})\right)^{2}+\gamma_{i}^{2}(\boldsymbol{p})}-\frac{1}{\left(\omega+\tilde{E}_{i}(\boldsymbol{p})\right)^{2}+\gamma_{i}^{2}(\boldsymbol{p})}\right)
$$

with $\tilde{E}_{i}^{2}(\boldsymbol{p})=\boldsymbol{p}^{2}+M_{i}^{2}(\boldsymbol{p})-\gamma_{i}^{2}(\boldsymbol{p})$ for $i \in[g, q, \bar{q}]$. These spectral functions (7) are antisymmetric in $\omega$ and normalized as

$$
\int_{-\infty}^{+\infty} \frac{d \omega}{2 \pi} \omega \rho_{i}(\omega, \boldsymbol{p})=\int_{0}^{+\infty} \frac{d \omega}{2 \pi} 2 \omega \rho_{i}(\omega, \boldsymbol{p})=1,
$$

where $M_{i}\left(T, \mu_{q}, \boldsymbol{p}\right), \gamma_{i}\left(T, \mu_{q}, \boldsymbol{p}\right)$ are the particle pole mass and width at finite three momentum $\boldsymbol{p}$, temperature $T$ and chemical potential $\mu_{q}$, respectively.

\subsection{The running coupling in $D Q P M^{*}$}

In contrast to the previous DQPM studies in Refs. 26 28 we report here a new solution for the determination of the effective coupling which is more flexible. The strategy to determine $g^{2}\left(T / T_{c}\right)$ is the following: For every temperature $T$ we fit the DQPM $^{*}$ entropy density $(1)$ to the entropy density $s^{l Q C D}$ obtained by lQCD. In practice, it has been checked that for a given value of $g^{2}$, the ratio $s\left(T, g^{2}\right) / T^{3}$ is almost constant for different temperatures and identical to $g^{2}$ in case of momentumindependent selfenergies, i.e. $\frac{\partial}{\partial T}\left(s\left(T, g^{2}\right) / T^{3}\right) \approx 0$. Therefore the entropy density $s$ and the dimensionless equation of state in the DQPM is a function of the effective coupling only, i.e. $s\left(T, g^{2}\right) / s_{S B}(T)=f\left(g^{2}\right)$. The functional form,

$$
f\left(g^{2}\right)=\frac{1}{\left(1+a_{1}\left(g^{2}\right)^{a_{2}}\right)^{a_{3}}},
$$

however, is also suited to describe $s^{l Q C D}\left(T, g^{2}\right) / s_{S B}$ in case of momentumdependent selfenergies in the DQPM* By inverting $f\left(g^{2}\right)$, one arrives at the following parametrization for $g^{2}$ as a function of $s / s_{S B}$ :

$$
g^{2}\left(s / s_{S B}, T\right) \sim\left(\frac{a}{T}+b\right)\left(\left(\frac{s / s_{S B}}{d(T)}\right)^{v(T)}-1\right)^{w(T)},
$$

with $s_{S B}=19 /\left(9 \pi^{2} T^{3}\right)$. Since the entropy density from lQCD has the proper high temperature limit, the effective coupling $g^{2}$ also gives the correct asymptotics for $T \rightarrow \infty$ and decreases as $g^{2} \sim 1 / \log \left(T^{2}\right)$. The temperature-dependent parameters $v(T), w(T)$ and $d(T)$ all have the functional form:

$$
f(T)=\frac{a}{\left(T^{b}+c\right)^{d}}(T+e),
$$

where the parameters $a, b, c, d$ and $e$ are fixed once for each function $v(T), w(T)$ and $d(T)$. 
Note that with the parametrization (9) for $g^{2}\left(s / s_{S B}, T\right)$ one can easily adapt to any equation of state and therefore avoid a refitting of the coupling in case of new (or improved) lattice data. However, the coupling 9 is valid only for a given number of quark flavors $N_{f}$ which is fixed by the lQCD equation of state.

To obtain $g^{2}\left(T / T_{c}\right)$ from $g^{2}\left(s / s_{S B}, T\right)$, we proceed as follows:

- Using the equation of state from the Wuppertal-Budapest collaboration, 20 which provide an analytical parametrization of the interaction measure $I / T^{4}$

$$
\frac{I(T)}{T^{4}}=\exp \left(-h_{1} / t-h_{2} / t^{2}\right) \cdot\left(h_{0}+\frac{f_{0}\left(\tanh \left(f_{1} \cdot t+f_{2}\right)+1\right)}{1+g_{1} \cdot t+g_{2} \cdot t^{2}}\right),
$$

with $t=T / 200 \mathrm{MeV}, h_{0}=0.1396, h_{1}=-0.18, h_{2}=0.035, f_{0}=2.76$, $f_{1}=6.79, f_{2}=-5.29, g_{1}=-0.47$ and $g_{2}=1.04$,

- we calculate the pressure $P / T^{4}$ by

$$
\frac{P(T)}{T^{4}}=\int_{0}^{T} \frac{I\left(T_{0}\right)}{T_{0}^{5}} d T_{0}
$$

- and then the entropy density

$$
s / s_{S B}=\frac{I(T) / T^{4}+4 P / T^{4}}{19 /\left(9 \pi^{2}\right)} .
$$

- Replacing $s / s_{S B}$ from Eq. (13) in Eq. (9) we obtain $g^{2}\left(T / T_{c}\right)$.

The procedure outlined above yields $g^{2}\left(T / T_{c}\right)$ for $\mu_{q}=0$. For finite $\mu_{q}$ we will make use of $g^{2}\left(T / T_{c}\right) \rightarrow g^{2}\left(T^{\star} / T_{c}\left(\mu_{q}\right)\right)$, with the $\mu_{q}$-dependent critical temperature $T_{c}\left(\mu_{q}\right)$ taken from Eq. (5). The running coupling (9)-(13) permits for an enhancement near $T_{c}$ as already introduced in Ref ${ }^{10}$

Figs. 1 (a)-(b) show the gluon and light quark masses and widths, respectively, at finite temperature and chemical potential for a momentum $p=1 \mathrm{GeV} / \mathrm{c}$. Furthermore, Fig. 1 (c) shows the gluon and light quark masses as a function of momentum (squared) $p^{2}$ at finite temperature $T=2 T_{c}$ and different $\mu_{q}$. Note that for $p=0$ we obtain higher values of the gluon and light quark masses (as a function of $T$ and $\mu_{q}$ ) since for finite momenta the masses decrease (at a given temperature and chemical potential), especially for the light quarks as seen in Fig 1 (c). The extension $T / T_{c} \rightarrow T^{\star} / T_{c}\left(\mu_{q}\right)$ for finite $\mu_{q}$ in the functional form for the strong coupling leads to lower values for the parton masses and widths at finite $\mu_{q}$ as compared to $\mu_{q}=0$ near $T_{c}\left(\mu_{q}\right)$.

An illustration of the actual spectral functions (in $\omega$ and momentum $p$ ) is given in Fig. 2 for a 'gluon' (l.h.s.) and a light 'quark' (r.h.s.) at temperature $T=200$ $\mathrm{MeV}$ for $\mu_{q}=0$. 

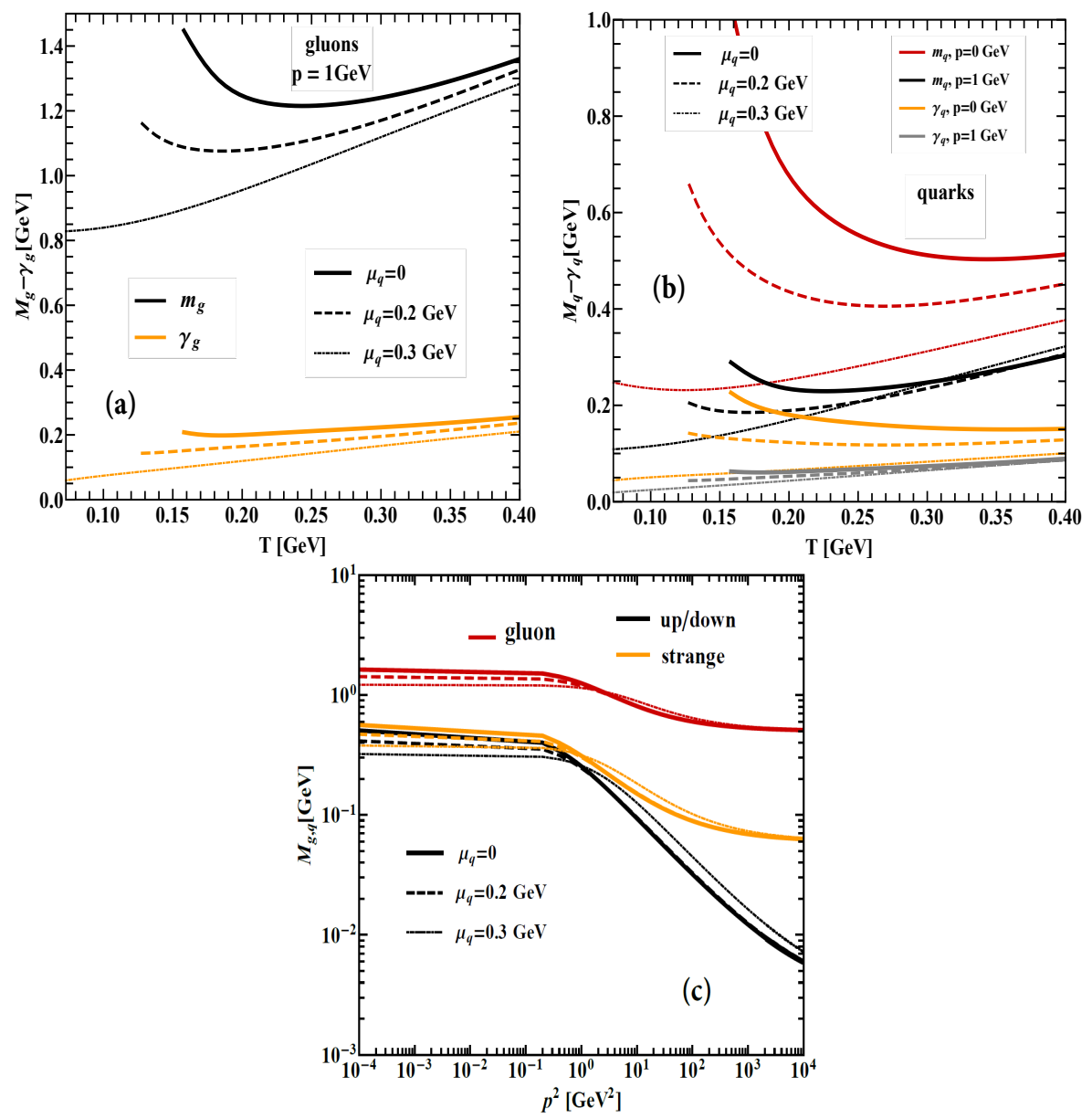

Fig. 1. (Color online) The DQPM* gluon (a) and light quark (b) masses and widths given by (3) using the coupling (9)-13) for different quark chemical potentials as a function of the temperature $T$. (c) Gluon and light quark masses as a function of the momentum squared for $T=2 T_{c}$ and $\mu_{q}=0,0.2,0.3 \mathrm{GeV}$. The figures are taken from Ref. $\frac{39}{39}$

\subsection{Thermodynamics of the $Q G P$ from $D Q P M^{*}$}

The expressions for the equation of state (energy density $\epsilon$, entropy density $s$ and pressure $P$ ) of strongly interacting matter have been given for finite temperature and chemical potential in Ref. ${ }^{19}$ for on-shell partons and in Ref! ${ }^{18}$ for the case of off-shell partons using the relations based on the stress-energy tensor $T^{\mu \nu}$. We recall that the approach for calculating the equation of state in the DQPM* is based on thermodynamic relations (see below). The procedure is as follows: One starts from the evaluation of the entropy density $s$ from (1) employing the masses and widths obtained from the expressions in Section 2.1. Then using the thermodynamic 

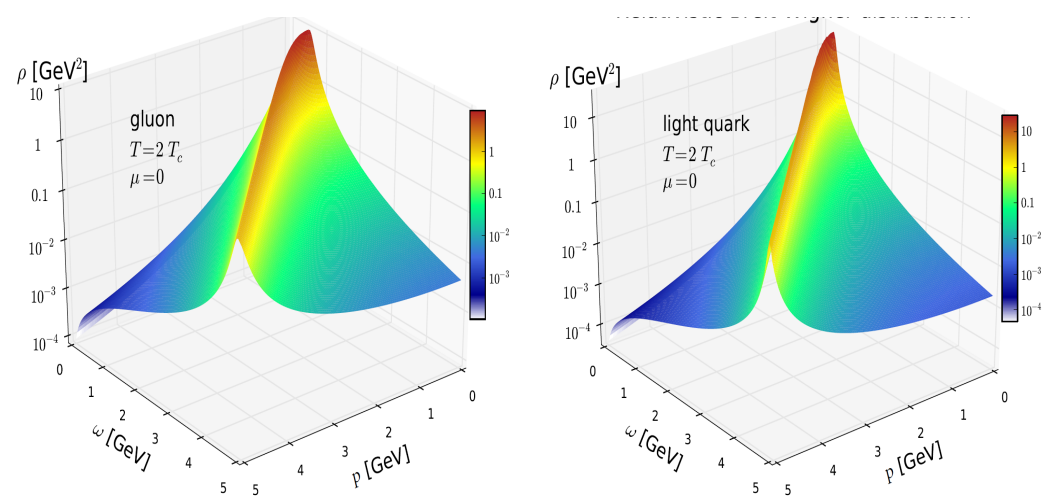

Fig. 2. (Color online) The DQPM* gluon (l.h.s.) and light quark (r.h.s.) spectral functions at $T$ $=200 \mathrm{MeV}$ as a function of momentum p and energy $\omega$.

relation $s=(\partial P / \partial T)_{\mu_{q}}$ (for a fixed quark chemical potential $\mu_{q}$ ) one obtains the pressure $P$ by integration of the entropy density $s$ over $T$ while the energy density $\epsilon$ can be gained using the relation,

$$
\epsilon\left(T, \mu_{B}\right)=T s\left(T, \mu_{B}\right)-P\left(T, \mu_{B}\right)+\mu_{B} n_{B}\left(T, \mu_{B}\right),
$$

where $n_{B}$ is the net baryon density.
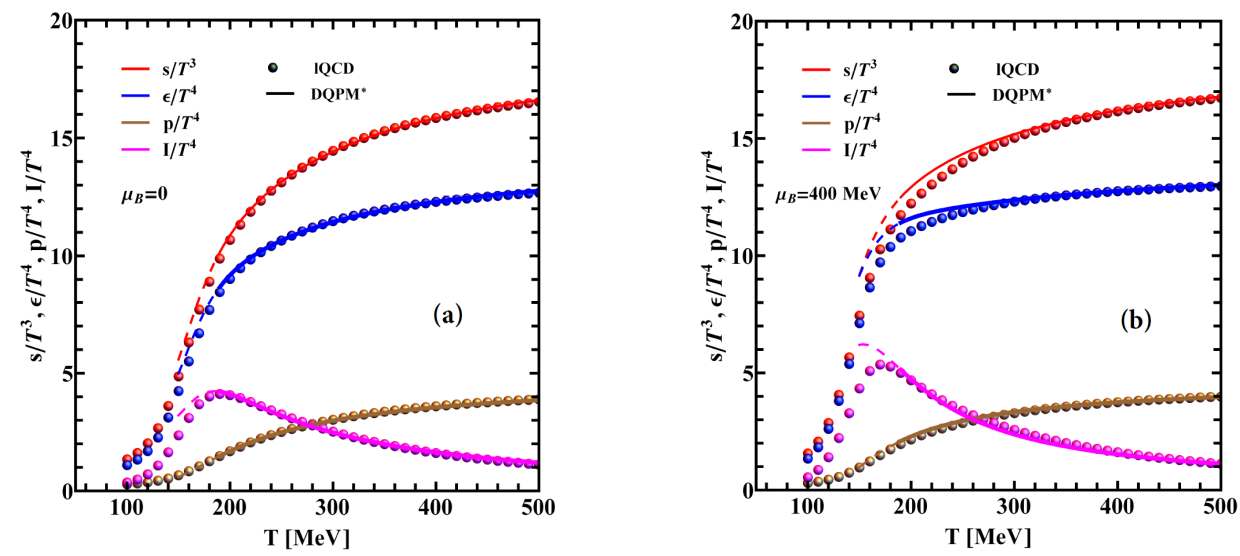

Fig. 3. (Color online) Scaled energy density $\epsilon$, entropy density s, pressure $P$ and trace anomaly $(I=\epsilon-3 P)$ as a function of temperature $T$ at $\mu_{B}=0$ (a) and at $\mu_{B}=400 \mathrm{MeV}$ (b) from $D Q P M^{*}$ compared to $l Q C D$ data from Ref!20 The figures are taken from Ref. ${ }^{[39}$

The energy density $\epsilon$, entropy density $s$, pressure $P$ and the interaction measure $\left[I\left(T, \mu_{q}\right)=\epsilon\left(T, \mu_{q}\right)-3 P\left(T, \mu_{q}\right)\right]$-known in lQCD as the trace anomaly- in the DQPM $^{*}$ are shown in Fig. 3 (a), (b) as a function of temperature $T$ for two values of 
the baryon chemical potential $\mu_{B}=0$ and $\mu_{B}=400 \mathrm{MeV}$, respectively (where $\mu_{B}=$ $3 \mu_{q}$ in our study). We, furthermore, compare our results with lattice calculations from Ref. ${ }^{20}$ and notice that our results are in a very good agreement with the lattice data for $\mu_{B}=0$ (a) and in case of $\mu_{B}=400 \mathrm{MeV}$ (b) for temperatures larger than $1.2 T_{c}\left(\mu_{q}\right)$. In the latter case we observe (for temperatures just above $T_{c}(\mu)$ ) some deviations which are expected to result from additional hadronic degrees of freedom in the crossover region. The small excess in quarks can be seen also in the net baryon density $n_{B}$, as we will show below.

At finite baryon chemical potential, i.e. $\mu_{B}=400 \mathrm{MeV}$, the maximum of the trace anomaly is shifted towards lower temperatures. We notice also the proper scaling of our DQPM* description of QGP thermodynamics, when moving from zero to finite quark chemical potential (cf. Fig 3 (a) and (b)).

\section{Quark number density and susceptibility from DQPM*}

\subsection{Baryon number density in the $D Q P M^{*}$}

The equation of state for vanishing chemical potential $\mu_{q}=0$ is defined solely by the entropy density $s$; for finite chemical potential $\mu_{q} \neq 0$ one has to include the particle density $n$. In the $\mathrm{DQPM}^{*}$ the quark density $n^{d q p}$ in the quasiparticle limit is defined in analogy to the entropy density (1) as, $\frac{40}{4}$

$$
\begin{aligned}
n^{d q p}= & -d_{q} \int \frac{d \omega}{2 \pi} \frac{d^{3} p}{(2 \pi)^{3}} \frac{\partial f_{F}\left(\left(\omega-\mu_{q}\right) / T\right)}{\partial \mu_{q}}\left(\Im \ln \left(-S_{q}^{-1}\right)+\Im \Sigma_{q} \Re S_{q}\right) \\
& -d_{\bar{q}} \int \frac{d \omega}{2 \pi} \frac{d^{3} p}{(2 \pi)^{3}} \frac{\partial f_{F}\left(\left(\omega+\mu_{q}\right) / T\right)}{\partial \mu_{q}}\left(\Im \ln \left(-S_{\bar{q}}^{-1}\right)+\Im \Sigma_{\bar{q}} \Re S_{\bar{q}}\right) .
\end{aligned}
$$

In case of the Lorentzian spectral function (7) the density $n^{d q p}$ in Eq. 15) can be split into the following two terms $n_{q}^{(0)}$ and $\Delta n_{q}$ as:

$$
\begin{aligned}
& n_{q}^{(0)}=d_{q} \int \frac{d^{3} p}{(2 \pi)^{3}} f_{q}^{(0)}-d_{\bar{q}} \int \frac{d^{3} p}{(2 \pi)^{3}} f_{\bar{q}}^{(0)}, \\
& \Delta n_{q}=\int \frac{d \omega}{(2 \pi)} \frac{d^{3} p}{(2 \pi)^{3}} \frac{\partial f_{q}\left(\left(\omega-\mu_{q}\right) / T\right)}{\partial \mu_{q}} \xi(\omega, p) \\
& +\int \frac{d \omega}{(2 \pi)} \frac{d^{3} p}{(2 \pi)^{3}} \frac{\partial f_{\bar{q}}\left(\left(\omega+\mu_{q}\right) / T\right)}{\partial \mu_{q}} \xi(\omega, p),
\end{aligned}
$$

with

$$
\xi(\omega, p)=\left(2 \gamma \omega \frac{\omega^{2}-\mathbf{p}^{2}-M^{2}}{\left(\omega^{2}-\mathbf{p}^{2}-M^{2}\right)^{2}+4 \gamma^{2} \omega^{2}}-\arctan \left(\frac{2 \gamma \omega}{\omega^{2}-\mathbf{p}^{2}-M^{2}}\right)\right)
$$

where $f_{q}^{(0)}=\left(\exp \left(\left(\sqrt{p^{2}+M^{2}}-\mu_{q}\right) / T\right)+1\right)^{-1}, f_{\bar{q}}^{(0)}=\left(\exp \left(\left(\sqrt{p^{2}+M^{2}}+\mu_{q}\right) / T\right)+\right.$ $1)^{-1}$ denote again the Fermi distribution functions for the on-shell quark and antiquark, with $M$ corresponding to the pole mass. 
Finally, note that the quark number density 15 follows from the same potential as the entropy density ${ }^{32}$ which ensures that it fulfills the thermodynamic relation $n=\left(\partial P / \partial \mu_{q}\right)_{T}$ (for fixed temperature $\mathrm{T}$ ). To be fully thermodynamically consistent the entropy and the particle density have to satisfy the Maxwell relation $(\partial n / \partial T)_{\mu_{q}}=\left(\partial s / \partial \mu_{q}\right)_{T}$. This provides further constraints on the effective coupling $g^{2}\left(T, \mu_{q}\right)$ at finite chemical potential which we neglect in the current approach. Nevertheless, it was checked that the violation of the latter Maxwell relation is generally small and most pronounced around $T_{c}$. We note, however, that when extending the approach to even larger chemical potentials the full thermodynamic consistency has to be taken into account. The baryon number density $n_{B}$, finally, is related to the quark number density by the simple relation $n_{B}=n^{d q p} / 3$.

\subsection{Susceptibilities in the $D Q P M^{*}$}

From the densities $n_{B}$ one may obtain other thermodynamic quantities like the pressure difference $\Delta P$ and the quark susceptibilities $\chi_{q}$, which can be confronted with lattice data for $N_{f}=2$ from Alton et al 41442 and for $N_{f}=3$ from Borsanyi et $a l{ }^{20}$ We recall that the quark-number susceptibility measures the static response of the quark number density to an infinitesimal variation of the quark chemical potential. From Eqs. (16)-(18) we calculate $\Delta P$ and $\chi_{q}$ as

$$
\begin{gathered}
\Delta P\left(T, \mu_{B}\right) \equiv P\left(T, \mu_{B}\right)-P(T, 0)=\int_{0}^{\mu_{B}} n_{B} d \mu_{B} ; \\
\chi_{q}(T)=\left.\frac{\partial n_{q}}{\partial \mu_{q}}\right|_{\mu_{q}=0} ; \quad \chi_{q}\left(T, \mu_{q}\right)=\frac{1}{9} \frac{\partial n_{B}}{\partial \mu_{B}} .
\end{gathered}
$$

Furthermore, for small $\mu_{q}$ a Taylor expansion of the pressure in $\mu_{q} / T$ can be performed which gives

$$
\frac{P\left(T, \mu_{q}\right)}{T^{4}}=\sum_{n=0}^{\infty} c_{n}(T)\left(\frac{\mu_{q}}{T}\right)^{n}, \quad c_{n}(T)=\left.\frac{1}{n !} \frac{\partial^{n}\left(P\left(T, \mu_{q}\right) / T^{4}\right)}{\partial\left(\mu_{q} / T\right)^{n}}\right|_{\mu_{q}=0},
$$

where $c_{n}(T)$ is vanishing for odd $n$ and $c_{0}(T)$ is given by $c_{0}(T)=P\left(T, \mu_{q}=0\right)$. As shown above the DQPM* compares well with lattice QCD results for $c_{0}(T)$. Since $\chi_{q}$ at finite $\mu_{q}$ is related to the pressure by

$$
\chi_{q}\left(T, \mu_{q}\right) / T^{2}=\partial^{2}\left(P / T^{4}\right) / \partial^{2}\left(\mu_{q} / T\right),
$$

one can define the susceptibility $\chi_{2}^{i j}$ at vanishing quark chemical potential as 20

$$
\frac{P\left(T, \mu_{i}\right)}{T^{4}}=\frac{P(T, 0)}{T^{4}}+\frac{1}{2} \sum_{i, j} \frac{\mu_{i} \mu_{j}}{T^{2}} \chi_{2}^{i j} \text {, with } \chi_{2}^{i j}=\left.\frac{1}{T^{2}} \frac{\partial n_{j}\left(T, \mu_{i}\right)}{\partial \mu_{i}}\right|_{\mu_{i}=\mu_{j}=0},
$$

which in case of 3 flavors with $\mu_{u}=\mu_{d}=\mu_{s}$ becomes

$$
\chi_{2}(T)=\left.\frac{1}{9} \frac{1}{T^{2}} \frac{\partial n_{q}\left(T, \mu_{q}\right)}{\partial \mu_{q}}\right|_{\mu_{q}=0}=\frac{1}{9} \frac{\chi_{q}(T)}{T^{2}} .
$$


We recall again that the susceptibilities are the central quantities in IQCD calculations for nonzero $\mu_{q}$.

\section{3. $n_{B}$ and $\chi_{q}: D Q P M^{*}$ vs $l Q C D$}

Using the masses and widths $(3)$ and the running coupling $(9)-(12)$, we calculate the baryon number density $n_{B}$ (16)-(17) and quark susceptibility $\chi_{2}$ including the finite width of the parton spectral functions. The results for $n_{B}$ and $\chi_{2}$ for $N_{f}=3$ are given in Fig. 4 (a) and (b), respectively. The comparison with the lattice data from Ref. ${ }^{20}$ is rather good which is essentially due to an extra contribution arising from the momentum dependence of the $\mathrm{DQPM}^{*}$ quasiparticles masses and widths. Such a momentum dependence in $m_{q, \bar{q}, g}$ and $\gamma_{q, \bar{q}, g}$ decreases the 'thermal average' of light quark and gluon masses which improves the description of 1QCD results for the susceptibilities. For comparison we also show the result for $\chi_{q}$ from the conventional DQPM, i.e. with momentum independent masses, which substantially underestimates the lattice data. The small difference between IQCD and DQPM* for $n_{B}$ and $\chi_{2}$ close to $T_{c}$ is related to a possible excess of light quarks and antiquarks which should combine to hadrons in the crossover region. We recall that the DQPM* describes only the QGP phase and deals with dynamical quarks and gluons solely.

Finally, we emphasize the challenge to describe simultaneously the entropy $s$ and pressure $P$ on one side and $n_{B}$ and $\chi_{2}$ on the other side. Indeed, increasing the light quark mass and width helps to improve the description of $s$ and $P$ (for $\mu_{B}=400 \mathrm{MeV}$ ), but this leads to a considerable decrease in $n_{B}$ and $\chi_{2}$. In other words, lighter quarks are favorable to improve the agreement with IQCD data on $n_{B}$ and $\chi_{2}$, however, this leads to an increase of $s$ and $P$, which can be only partially counterbalanced by an increasing gluon mass and width (which do not enter $n_{b}$ and $\left.\chi_{2}\right)$.

\section{Transport properties of the hot QGP from DQPM*}

\subsection{Shear and bulk viscosities}

In this Section we focus on the transport coefficients of the QGP using the relaxation time approximation (RTA). In the dilute gas approximation the relaxation time $\tau_{i}$ of the particle $i$ is obtained for on- or off-shell quasi-particles by means of the partonic scattering cross sections, where the $q q, q \bar{q}, q g$ and $g g$ elastic scattering processes as well as some inelastic processes involving chemical equilibration, such as $g g \rightarrow q \bar{q}$ are included in the computation of $\tau_{i} \cdot 27$ For the DQPM* approach we do not need the explicit cross sections since the inherent quasi-particle width $\gamma_{i}\left(T, \mu_{q}, p\right)$ directly provides the total interaction rate ${ }^{[18}$ To this end we only have to evaluate the average of the momentum dependent widths $\gamma_{g}\left(T, \mu_{q}, p\right)$ and $\gamma_{q}\left(T, \mu_{q}, p\right)$ over the thermal distributions at fixed $T$ and $\mu_{q}$, i.e. $\bar{\gamma}_{g}\left(T, \mu_{q}\right)$ and $\bar{\gamma}_{q}\left(T, \mu_{q}\right)$.

The shear viscosity $\eta\left(T, \mu_{q}\right)$ is defined in the dilute gas approximation for the 

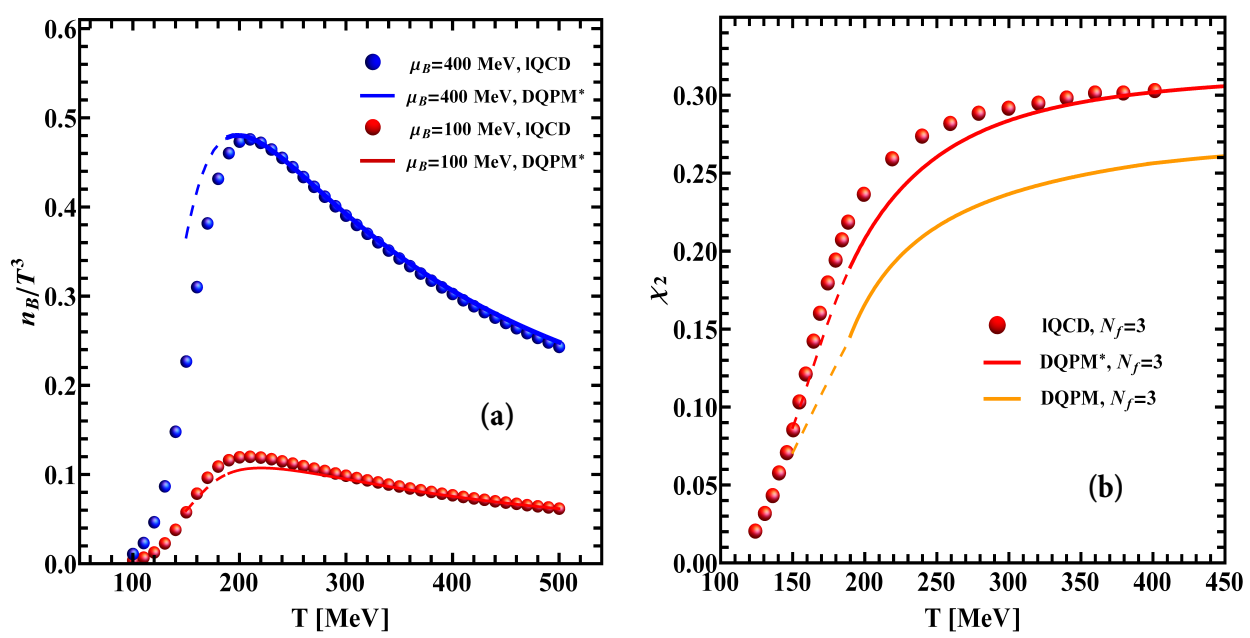

Fig. 4. (Color online) (a) The baryon number density $n_{B} / T^{3}$ from $D Q P M^{*}$ as compared to lattice data from Ref.20 for $N_{f}=3$ for a quark chemical potential $\mu_{q}=0$. (b) The susceptibility $\chi_{2}$ from $D Q P M^{*}$ as compared to lattice data from Ref ${ }^{[20}$ for $N_{f}=3$ and $\mu_{q}=0$ using Eq. (23). The lower (orange) line gives the result from the conventional DQPM, i.e. with momentum independent masses. The figures are taken from Ref.39

case of the $\mathrm{DQPM}^{*}$ off-shell particles by $2 7 \longdiv { 4 3 }$

$$
\begin{aligned}
\eta\left(T, \mu_{q}\right)= & \frac{1}{15 T} d_{g} \int \frac{d^{3} p}{(2 \pi)^{3}} \int \frac{d \omega}{2 \pi} \omega \bar{\tau}_{g}\left(T, \mu_{q}\right) f_{g}(\omega / T) \times \rho_{g}(\omega, \boldsymbol{p}) \frac{\boldsymbol{p}^{4}}{\omega^{2}} \Theta\left(P^{2}\right) \\
+ & \frac{1}{15 T} \frac{d_{q}}{6} \int \frac{d^{3} p}{(2 \pi)^{3}} \int \frac{d \omega}{2 \pi} \omega\left[\sum_{q}^{u, d, s} \bar{\tau}_{q}\left(T, \mu_{q}\right) f_{q}\left(\left(\omega-\mu_{q}\right) / T\right) \rho_{q}(\omega, \boldsymbol{p})\right. \\
& \left.+\sum_{\bar{q}}^{\bar{u}, \bar{d}, \bar{s}} \bar{\tau}_{\bar{q}}\left(T, \mu_{q}\right) f_{\bar{q}}\left(\left(\omega+\mu_{q}\right) / T\right) \rho_{\bar{q}}(\omega, \boldsymbol{p})\right] \frac{\boldsymbol{p}^{4}}{\omega^{2}} \Theta\left(P^{2}\right),
\end{aligned}
$$

where $\boldsymbol{p}$ is the three-momentum and $P^{2}$ the invariant mass squared. The functions $\rho_{g}, \rho_{q}, \rho_{\bar{q}}$ stand for the gluon, quark and antiquark spectral functions, respectively, and $f_{q}\left(f_{\bar{q}}\right)$ stand for the equilibrium distribution functions for particle and antiparticle. The medium-dependent relaxation times $\bar{\tau}_{q, g}\left(T, \mu_{q}\right)$ in (24) are given in the $\mathrm{DQPM}^{*}$ by:

$$
\bar{\tau}_{q, g}\left(T, \mu_{q}\right)=\left(\bar{\gamma}_{q, g}\right)^{-1}\left(T, \mu_{q}\right)
$$

with:

$$
\begin{aligned}
& \bar{\gamma}_{q, g}\left(T, \mu_{q}\right)=\left\langle\gamma_{q, g}\left(T, \mu_{q}, p\right)\right\rangle_{p} \\
& =\left(n_{q, g}^{\text {off }}\left(T, \mu_{q}\right)\right)^{-1} \times \int \frac{d^{3} p}{(2 \pi)^{3}} \frac{d \omega}{(2 \pi)} \omega \gamma_{q, g}\left(T, \mu_{q}, p\right) \rho_{f}(\omega) f_{q, g}\left(\omega, T, \mu_{q}\right) \Theta\left(P^{2}\right),
\end{aligned}
$$


where

$$
n_{f, g}^{\mathrm{off}}\left(T, \mu_{q}\right)=\int \frac{d^{3} p}{(2 \pi)^{3}} \frac{d \omega}{(2 \pi)} \omega \rho_{f}(\omega) f_{f, g}\left(\omega, T, \mu_{q}\right) \Theta\left(P^{2}\right)
$$

denotes the off-shell density of quarks, antiquarks or gluons. We note in passing that the shear viscosity $\eta$ can also be computed using the stress-energy tensor and the Green-Kubo formalism. ${ }^{25]}$ However, explicit comparisons of both methods in Ref ${ }^{25}$ have shown that the solutions are rather close. This holds especially for the case of the scattering of massive partons where the transport cross section is not very different from the total cross section as also pointed out in Ref. ${ }^{44}$ Furthermore, we mention that the definition of the shear viscosity $\eta$ is strictly valid only in the on-shell limit, however, can be employed also in the DQPM* since the relaxation times $\bar{\tau}_{i}$ do not depend on the masses.

We show the DQPM* results for $\eta / s$, where $s$ is the DQPM* entropy density, in Fig 5 (a) as a function of the temperature. The (upper) orange solid line represents the case of the standard DQPM where the parton masses and widths are independent of momenta as calculated in Ref. ${ }^{27}$ The thick red solid line displays the result using Eqs. (24) and (25), where the parton masses and width are temperature, chemical potential and momentum dependent. Finally, the black solid line refers to the calculation of $\eta / s$ in Yang-Mills theory from the Kubo formula using an exact diagrammatic representation in terms of full propagators and vertices from Ref. 45

Fig. 5 (a) shows that $\eta / s$ from $D^{2} \mathrm{PPM}^{*}$ is in the range of the 1QCD data and significantly lower than the pQCD limit. As a function of temperature $\eta / s$ shows a minimum around $T_{c}$, similar to atomic and molecular systems $\frac{46}{4}$ and then increases slowly for higher temperatures. This behavior is very much the same as in the standard DQPM (upper orange line) as shown in Ref.25 Therefore, the produced QGP shows features of a strongly interacting fluid unlike a weakly interacting parton gas as had been expected from perturbative QCD (pQCD). The minimum of $\eta / s$ at $T_{c}=158 \mathrm{MeV}$ is close to the lower bound of a perfect fluid with $\eta / s=1 /(4 \pi) 4748$ for infinitely coupled supersymmetric Yang-Mills gauge theory (based on the AdS/CFT duality conjecture). This suggests the "hot QCD matter" to be the "most perfect fluid" 46 Furthermore, the ratio $\eta / s$ in DQPM* is slightly larger than in the pure gluonic system (solid black line) due to a lower interaction rate of quarks and antiquarks relative to gluons.

The explicit dependencies of $\eta / s$ on $T$ and $\mu_{q}$ are shown in Fig. 5 (b) where $\eta / s$ is seen to increase smoothly for finite but small $\mu_{q}$. We point out again that extrapolations to larger $\mu_{q}$ become increasingly uncertain.

The bulk viscosity (defined in Ref. ${ }^{43}$ for the on-shell case) reads in the relaxation 

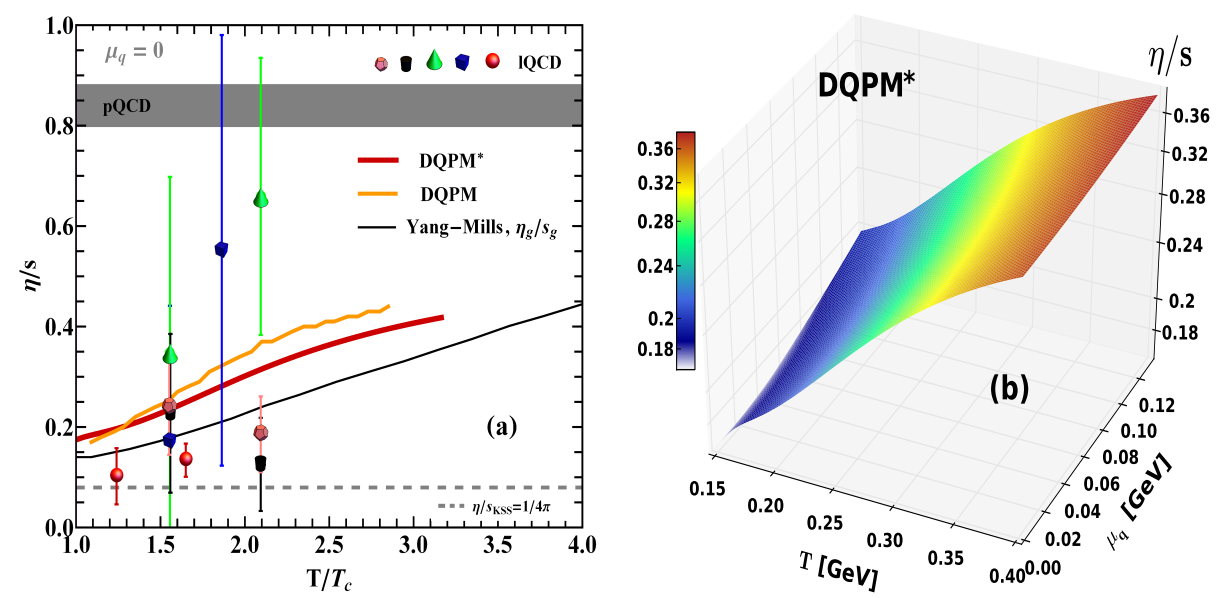

Fig. 5. (Color online) The shear viscosity to entropy density ratio $\eta / s$ from different models as a function of temperature $T$ for $\mu_{q}=0$ (a) and $\eta / s$ given by the $D Q P M^{*}$ approach as a function of $\left(T, \mu_{q}\right)(b)$. The orange solid line in (a) results from the standard DQPM where the parton masses and widths are independent of momenta 27 The thick red solid line shows the DQPM* result using Eqs. (24) and (25), where the parton masses and width are temperature, chemical potential and momentum dependent. The lattice QCD data for pure SU(3) gauge theory are taken from Ref $\lceil 99$ (red spheres), from Ref!50 (green pyramid and blue cubic), and from Ref! 51 (black cylinder and pink penthagone). The orange dashed line gives the Kovtun-Son-Starinets lower bouna $4748(\eta / s)_{K S S}=1 /(4 \pi)$. Finally, the black solid line refers to the calculation of $\eta / s$ in Yang-Mills theory from Ref 45 The figures are taken from Ref! 39

time approximation (RTA) for the case of off-shell DQPM* partons as:

$$
\begin{aligned}
\zeta\left(T, \mu_{q}\right)= & \frac{1}{9 T} d_{g} \int \frac{d^{3} p}{(2 \pi)^{3}} \int \frac{d \omega}{2 \pi} \omega \bar{\tau}_{g}\left(T, \mu_{q}\right) f_{g}(\omega / T) \rho_{g}(\omega, \boldsymbol{p}) \Theta\left(P^{2}\right) \frac{1}{\omega^{2}} F_{g}(\omega, \boldsymbol{p}) \\
& +\frac{1}{9 T} \frac{d_{q}}{6} \int \frac{d^{3} p}{(2 \pi)^{3}} \int \frac{d \omega}{2 \pi} \omega\left[\sum_{q}^{u, d, s} \bar{\tau}_{q}\left(T, \mu_{q}\right) f_{q}\left(\left(\omega-\mu_{q}\right) / T\right) \rho_{q}(\omega, \boldsymbol{p})\right. \\
& \left.+\sum_{\bar{q}}^{\bar{u}, \bar{d}, \bar{s}} \bar{\tau}_{\bar{q}}\left(T, \mu_{q}\right) f_{\bar{q}}\left(\left(\omega+\mu_{q}\right) / T\right) \rho_{\bar{q}}(\omega, \boldsymbol{p})\right] \Theta\left(P^{2}\right) \frac{1}{\omega^{2}} F_{q}(\omega, \boldsymbol{p})
\end{aligned}
$$

with

$$
F_{i}(\omega, \boldsymbol{p})\left[\boldsymbol{p}^{2}-3 c_{s}^{2}\left(\omega^{2}-T^{2} \frac{d M_{i}^{2}}{d T^{2}}\right)\right]^{2}
$$

and essentially depends on the mass derivatives $\partial M_{i}^{2} / \partial T^{2}$, the temperature $T$, and the speed of sound squared $c_{s}^{2}$. All these quantities are accessable within the $\mathrm{DQPM}^{*}$ such that the results for the bulk viscosity again do not imply any new parameter.

The bulk viscosity (divided by the entropy density $s$ ) from the $\mathrm{DQPM}^{*}$ is displayed in Fig. 6 (a) and shows a very different temperature dependence than $\eta / s$. 
Indeed, for high temperatures we find the limit $\zeta / s \rightarrow 0$. Moreover, the behavior around $T_{c}$ shows a peak in lQCD as well as in the DQPM and DQPM* which is essentially due to the derivative $\partial M_{i}^{2} / \partial T^{2}$ in Eq. 27). Accordingly, the infrared enhancement in the DQPM* masses is mandatory to achieve a maximum in the bulk viscosity $\zeta\left(T, \mu_{q}\right)$ to entropy ratio $\zeta / s$ close to $T_{c}$ in line with lQCD. This enhancement close to $T_{c}$ is lower in the DQPM* as in the DQPM probably due to a lower infrared enhancement in the coupling squared. Note, however, that such an enhancement does not show up in the NJL calculations for $\zeta / s$ from Ref ${ }^{[19}$ (black solid line in (a)). The explicit dependencies of $\zeta / s$ on $T$ and $\mu_{q}$ from the DQPM* are shown in Fig. 6 (b).
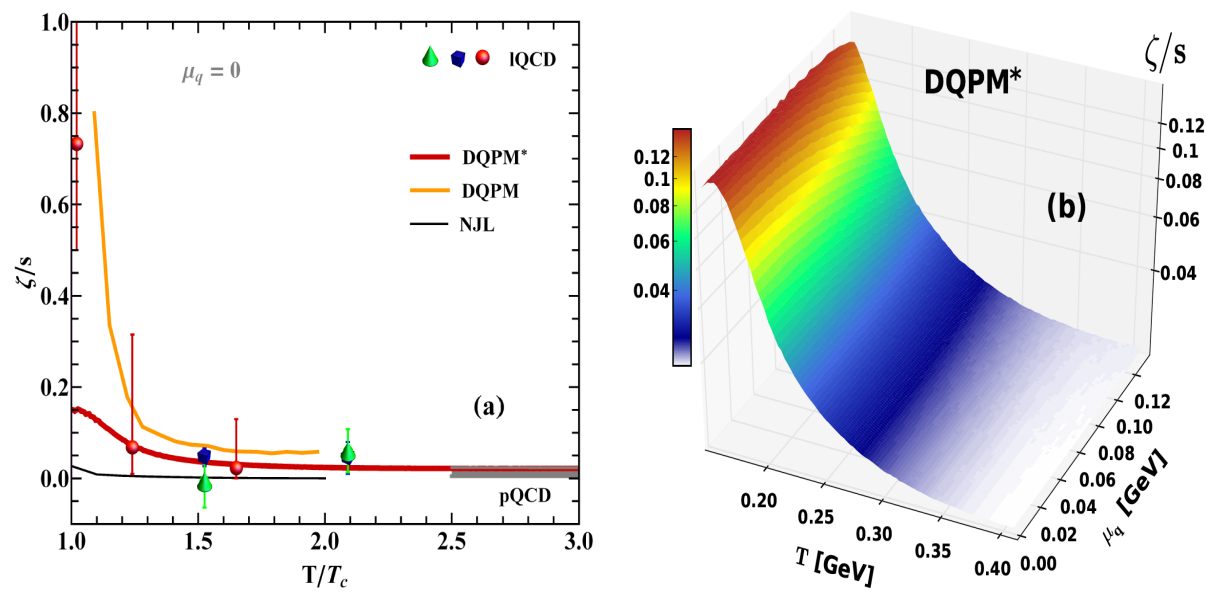

Fig. 6. (Color online) The bulk viscosity to entropy density ratio $\zeta / s$ from $D Q P M^{*}$ as a function of temperature $T$ for $\mu_{q}=0$ (a) and $\zeta / s$ given by the $D Q P M^{*}$ approach as a function of $T$ and $\mu_{q}$ (b). The orange solid line in (a) results from the standard DQPM where the parton masses and widths are independent of momenta 27 The lattice $Q C D$ data points for pure $S U(3)$ gauge theory are taken from Ref $f^{[49}$ (red spheres), 51 (blue cubic) and from Ref ${ }^{[50}$ (green pyramid). Finally, the black solid line in (a) refers to the calculation of $\zeta / s$ from the Nambu-Jona-Lasinio model for $S U(3)_{f}$ from Ref! 19

\subsection{Electric conductivity}

Whereas the shear and bulk viscosities depend on the properties of quarks, antiquarks and gluons the electric conductivity $\sigma_{e}$ only depends on electrically charged quarks and antiquarks and thus provides independent information on the response of the QGP to external electric fields $52[53$ It probes exclusively the fermion properties (as in case of the quark susceptibilities) and the interaction strength with gluons enters only indirectly via the total width of the quarks and antiquarks. The electric conductivity $\sigma_{e}$ is also important for the creation of electromagnetic fields in ultra-relativistic nucleus-nucleus collisions from partonic degrees-of-freedom, since 
$\sigma_{e}$ specifies the imaginary part of the electromagnetic (retarded) propagator and leads to an exponential decay of the propagator in time $\sim \exp \left(-\sigma_{e}\left(t-t^{\prime}\right)\right)$. Furthermore, $\sigma_{e}$ also controls the photon spectrum in the long wavelength limit 54

We recall that the dimensionless ratio $\sigma_{e} / T$ in the quasiparticle approach is given by the relativistic Drude formula $\sqrt{19}|52| 53$

$$
\sigma_{e}\left(T, \mu_{q}\right)=\sum_{f, \bar{f}}^{u, d, s} \frac{e_{f}^{2} n_{f}^{\text {off }}\left(T, \mu_{q}\right)}{\bar{\omega}_{f}\left(T, \mu_{q}\right) \bar{\gamma}_{f}\left(T, \mu_{q}\right)},
$$

with

$$
\bar{\omega}_{f}\left(T, \mu_{q}\right)=\left(n_{f}^{\text {off }}\left(T, \mu_{q}\right)\right)^{-1} \int \frac{d^{3} p}{(2 \pi)^{3}} \frac{d \omega}{(2 \pi)} \omega^{2} \rho_{f}(\omega, \boldsymbol{p}) f_{f}\left(\left(\omega \pm \mu_{q}\right) / T\right),
$$

where the quantity $\bar{\omega}_{q}\left(T, \mu_{q}\right)$ is the quark (antiquark) energy averaged over the equilibrium distributions at finite $T$ and $\mu_{q}$ while $\bar{\gamma}_{q}\left(T, \mu_{q}\right)$ is the averaged quark width, as given in Eq. (26).

The actual results for $\sigma_{e} / T$ are displayed in Fig. 7 (a) in terms of the thick red solid line in comparison to recent $1 \mathrm{QCD}$ data from Refs 55 and the result from previous studies within the DQPM ${ }^{19}$ (thin orange line). Again we find a minimum in the partonic phase close to $T_{c}$ and a rise with the temperature $T$. The explicit dependencies of $\sigma_{e} / T$ on $T$ and $\mu_{q}$, shown in Fig. 77(b), is also increasing smoothly for finite but small $\mu_{q}$. We finally note that the lower values for $\sigma_{e} / T$ in the DQPM* relative to the DQPM result from using the relativistic Drude formula 29 instead of its nonrelativistic counterpart.

\section{Differential partonic cross sections and transport coefficients for charm quarks in the hot medium}

Since the properties of charm degrees of freedom $Q$ cannot be determined from the usual thermodynamic quantities due to their large mass (and low occupation probability in thermal equilibrium) we calculate the charm interaction rates in the partonic medium dynamically via explicit cross sections. We will use the standard DQPM with momentum-independent selfenergies to evaluate the charm scattering with the 'dressed' $(u, d, s)$ partons.

\section{1. $q Q$ and $g Q$ elastic scattering at finite $T$ and $\mu_{q}$}

The process $q Q \rightarrow q Q$ is calculated here to lowest order in the perturbation expansion using the extended Feynman rules for massless quarks in Politzer's review 64 for the case of finite masses and widths. The color sums are evaluated using the techniques discussed in Ref. ${ }^{64}$; the spin sums will be discussed below. Contrary to the case of massless gluons where the "Transverse gauge" is used, the "Lorentz covariance" is used for the case of massive gluons here since a finite mass in the gluon propagator allows to fix the 0 'th components of the gluon fields $A_{a}^{0}(a=1, \cdots, 8)$ 

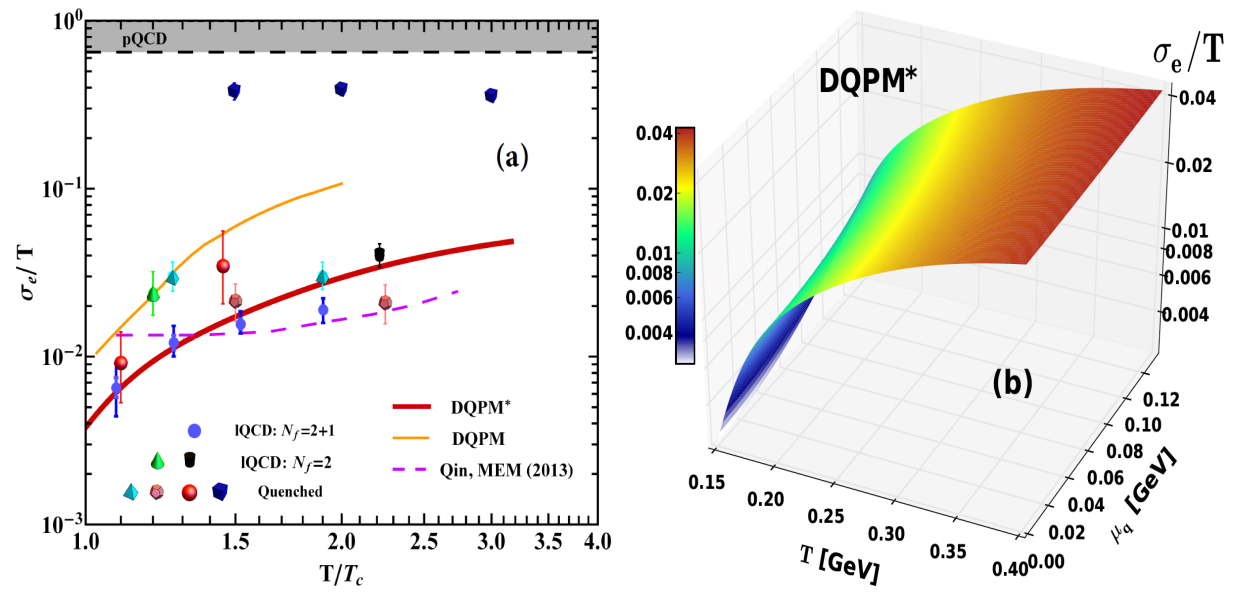

Fig. 7. (Color online) The ratio $\sigma_{e} / T$ from different models as a function of temperature $T$ for $\mu_{q}=0$ (a) and $\sigma_{e} / T$ given by the $D Q P M^{\star}$ approach as a function of $\left(T, \mu_{q}\right)(b)$. The orange thin solid line in (a) results from the standard DQPM where the parton masses and widths are independent of momenta 27 The red thick solid line shows the DQPM* result using Eqs. 29), where the parton masses and width are temperature, chemical potential and momentum dependent. The lattice QCD data are taken from Ref.55 (red spheres), Ref.56 (pink pentagon), Ref 57 (blue cubic), Ref!58 (Cyan pyramid), Ref!59 (green cone), Ref![60 (black cylinder), Ref! [61 (blue disk). Qin, MEM (2013) refers to Ref!62 where a Dyson-Schwinger approach is used. The electric charge is explicitly multiplied out using $e^{2} \approx 4 \pi / 137$. The average charge squared is $C_{E M}=8 \pi \alpha / 3$ with $\alpha \approx 1 / 137$. Note that the $p Q C D$ result at leading order beyond the leading

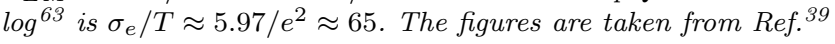

by the spatial degrees of freedom $A_{a}^{k}(k=1,2,3)$. Furthermore, the divergence encountered in the $t$-channel (Refs. 65 67 ) - when calculating the total cross sections $\sigma^{q Q}$ and $\sigma^{g Q}$ - is cured self-consistently since the infrared regulator is given by the finite DQPM gluon mass (and width). In our calculations, we have developed two different models, the so-called DpQCD (Dressed pQCD) and IEHTL (Infrared Enhanced HTL) model. In the first we consider only massive gluons and light and heavy quarks with masses given by the DQPM pole masses, whereas both the DQPM masses and widths are considered in the IEHTL model ${ }^{28}$ In the following we will only report on results obtained within the DpQCD since a finite width in the charm and light quark spectral functions was shown to lead to very moderate modifications of the results ${ }^{28}$

The elementary Feynman diagrams for the $q Q$ and $g Q$ elastic scattering at order $O\left(\alpha_{s}\right)$ are illustrated in Fig. 8 .

For on-shell $q Q$ elastic scattering, the $t$-channel invariant squared amplitude averaged over the initial spin and color degrees of freedom and summed over the final state spin and color $-\mathcal{M}_{t}$ is given by

$$
\sum\left|\mathcal{M}_{t}\right|^{2}=\frac{4 g^{4}}{9\left(t-m_{g}^{2}\right)^{2}}\left[\left(s-M_{Q}^{2}-m_{q}^{2}\right)^{2}+\left(u-M_{Q}^{2}-m_{q}^{2}\right)^{2}+2\left(M_{Q}^{2}+m_{q}^{2}\right) t\right],
$$



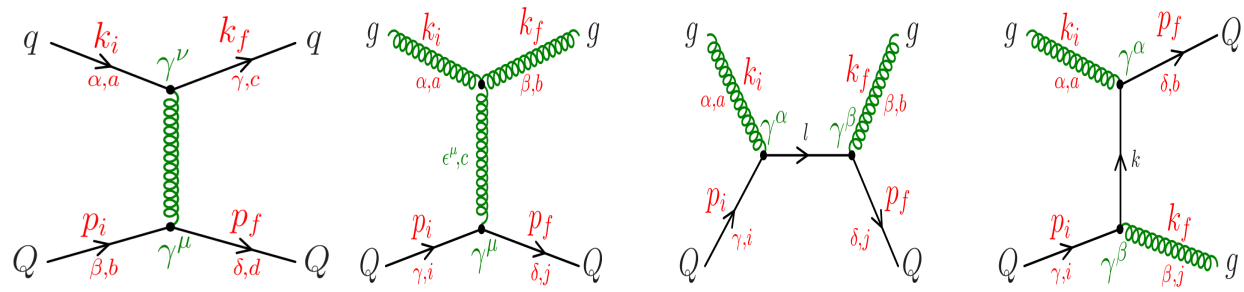

Fig. 8. (Color online) Feynman diagrams for the $q Q \rightarrow q Q$ and $g Q \rightarrow g Q$ scattering processes. Latin (Greek) subscripts denote colour (spin) indices. $k_{i}$, resp. $p_{i}\left(k_{f}\right.$, resp. $\left.p_{f}\right)$ denote the initial (final) 4-momentum of the light quark or the gluon, resp. the heavy quark $Q$. The invariant energy squared is given by $s=\left(p_{i}+k_{i}\right)^{2}, t=\left(p_{i}-p_{f}\right)^{2}$, and $u=\left(p_{i}-k_{f}\right)^{2}$. The figure is taken from Ref!28

where $m_{q}\left(M_{Q}\right)$ is the light quark (heavy quark) mass and $m_{g}$ is the DQPM exchanged gluon mass.

In the off-shell picture we take into account not only the finite masses of the partons, but also their spectral functions, i.e. their finite widths. Since the light quark and heavy quark masses change before and after the scattering ('quasi-elastic' process) we introduce the mass $m_{q}^{i}$ for the initial $q$ and $m_{q}^{f}$ for the final $q$, and allow for different masses of the heavy quark, $M_{Q}^{i}$ for the initial $Q$ and $M_{Q}^{f}$ for the final $Q$. The squared amplitude - averaged over the initial spin and color degrees of freedom and summed over the final state spin and color - gives:

$$
\begin{aligned}
\sum|\mathcal{M}|^{2}= & \frac{2 g^{4}}{9\left[\left(t-m_{g}^{2}\right)^{2}+4 \gamma_{g}^{2} q_{0}^{2}\right]} \times \\
& {\left[4\left(p_{f}^{\mu} p_{i}^{\nu}+p_{i}^{\mu} p_{f}^{\nu}+g^{\mu \nu} \frac{t}{2}\right)\right]\left[4\left(k_{f, \mu} k_{i, \nu}+k_{i, \mu} k_{f, \nu}+g_{\mu \nu} \frac{t}{2}\right)\right], }
\end{aligned}
$$

where we have incorporated the DQPM propagators (i.e. $t_{ \pm}^{*}=t-m_{g}^{2} \pm 2 i \gamma_{g} q_{0}$, where $m_{g}, \gamma_{g}$ are, respectively, the effective gluon mass and total width at temperature $T$ and quark chemical potential $\mu_{q}$ while $q^{0}=p_{f}^{0}-p_{i}^{0}=k_{f}^{0}-k_{i}^{0}$ is the gluon energy in the $t$-channel). Thus the divergence in the gluon propagator in the $t$-channel is regularized.

The relative contribution of the off-shell partons to the pQCD cross section is expected to change due to different kinematical thresholds and to the changes in the matrix element- corresponding to the diagram in Fig. 8. The off-shell kinematical limits for the momentum-transfer squared $t$ and the expressions of the Mandelstam variables in the case of off-shell heavy quark scattering are given in Ref ${ }^{26}$ Inspite of these expectations the actual results in Ref. ${ }^{28}$ show that the finite width of the partons has only a minor impact on the charm scattering with the bulk partons.

Figs. 9 (a) and (b) show explicitly the temperature and $\sqrt{s}$ dependences of the $u c$ and $g c$ elastic cross sections at $\mu_{q}=0$, as described in the DpQCD approach. We find that an increasing medium temperature $T$ leads to an increase of the 
thermal gluon mass (infrared regulator) and hence to a decrease of the DpQCD $u c$ and $g c$ elastic cross sections. We recall that the effective gluon mass is roughly proportional to $T$ for temperatures above $0.2 \mathrm{GeV}$. The large enhancement of the total cross section for temperatures close to $T_{c}\left(\mu_{q}\right)$, furthermore, can be traced back to the infrared enhanced coupling.
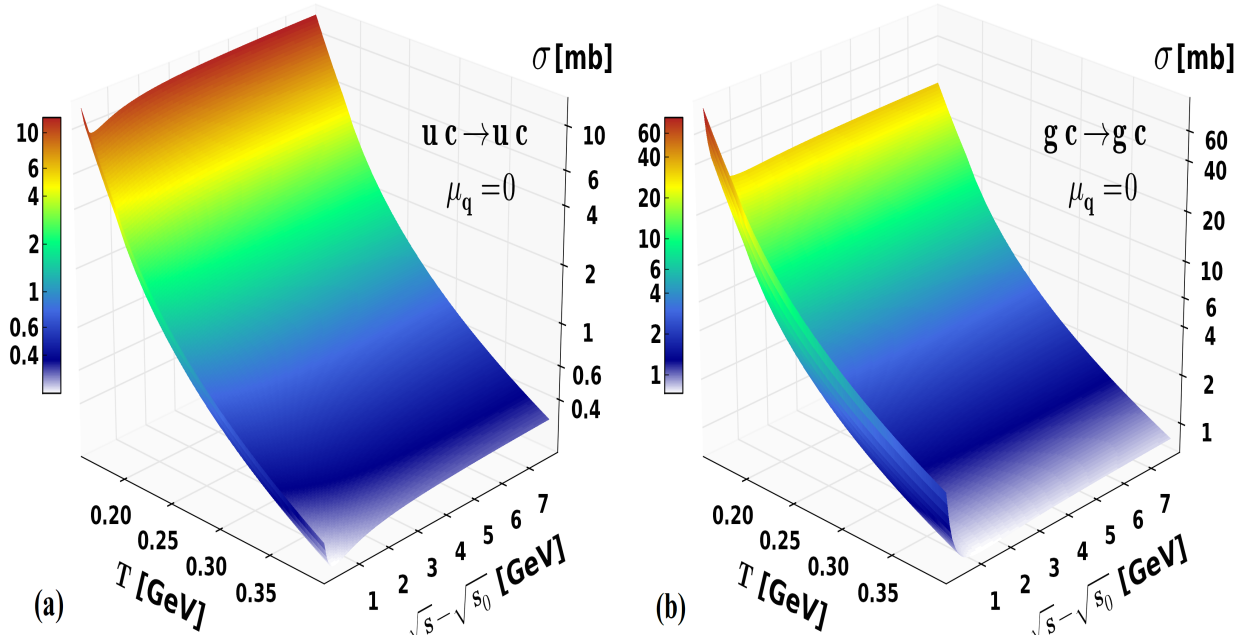

Fig. 9. (Color online) Elastic cross section of $u c \rightarrow u c(a)$ and $g c \rightarrow g c$ (b) scattering as a function of the temperature $T$ and the invariant energy above threshold $\sqrt{s}-\sqrt{s_{0}}$, where $\sqrt{s_{0}}$ is the threshold energy, for on-shell partons as described by the DpQCD approach at $\mu_{q}=0$. The figures are taken from Ref? 28

\subsection{Heavy quark interaction rates in a medium at finite $T$ and $\mu_{q}$}

Using the elastic cross section for $q(\bar{q}) Q$ and $g Q$ collisions, for on- and off-shell partons - as calculated in Sec. 5.1 - we evaluate the interaction rate of a heavy quark $Q$ with momentum $\boldsymbol{p}$ and energy $E$ propagating through a QGP in thermal and chemical equilibrium at a given temperature $T$ and quark chemical potential $\mu_{q}$. The occupation numbers of the light quarks/antiquarks of the plasma are described by a Fermi-Dirac distribution $f_{q, \bar{q}}(\boldsymbol{q})=\left(e^{\left(E_{q} \mp \mu_{q}\right) / T}+1\right)^{-1}$ whereas the gluons follow a Bose-Einstein distribution $f_{g}(\boldsymbol{q})=\left(e^{E_{g} / T}-1\right)^{-1}$.

For on-shell particles (DpQCD) and in the reference system in which the heavy quark has the velocity $\boldsymbol{\beta}=\boldsymbol{p} / E$ the (on-shell) interaction rate $R^{\mathrm{on}}(\boldsymbol{p})=d N_{\text {coll }}^{2} \overrightarrow{\mathrm{l}}^{2} / d t$ for $2 \rightarrow 2$ collisions is given by, 68

$$
R^{\mathrm{on}}\left(\boldsymbol{p}, T, \mu_{q}\right)=\sum_{q, \bar{q}, g} \frac{M_{Q}}{16(2 \pi)^{4} E} \int \frac{q^{3} m_{0}^{\mathrm{on}}(s) f_{r}(\boldsymbol{q})}{s E_{q}} d q
$$


where the sum is over the light quarks/antiquarks and gluons of the medium. In Eq. (33) $f_{r}(\boldsymbol{q})$ is the invariant distribution of the plasma constituents in the rest frame of the heavy quark, given for the quark/antiquark by:

$$
\int d \Omega f_{r}(\boldsymbol{q})=2 \pi \int_{-1}^{1} d \cos \theta_{r} \frac{1}{e^{\left(u^{0} E_{q}-u q \cos \theta_{r} \mp \mu_{q}\right) / T}+1},
$$

with $u \equiv\left(u^{0}, \boldsymbol{u}\right)=\frac{1}{M_{Q}}(E,-\boldsymbol{p})$ being the fluid 4-velocity measured in the heavyquark rest frame, while $\theta_{r}$ is the angle between $\boldsymbol{q}$ and $\boldsymbol{u}$. The quantity $m_{0}^{\text {on }}(s)$ in Eq. (33) is related to the transition amplitude $\left|\mathcal{M}_{2,2}\right|^{2}$ of the collision $q(\bar{q}, g) Q \rightarrow$ $q(\bar{q}, g) Q$ by

$$
m_{0}^{\mathrm{on}}(s)=\frac{1}{2 p_{c m}^{2}(s)} \int_{-4 p_{c m}^{2}}^{0} \frac{1}{g_{Q} g_{p}} \sum_{i, j} \sum_{k, l}\left|\mathcal{M}_{2,2}(s, t ; i, j \mid k, l)\right|^{2} d t,
$$

with $p_{c m}=\left(q M_{Q}\right) / \sqrt{s}$ denoting the momentum of the scattering partners in the c.m. frame and $g_{Q}\left(g_{p}\right)$ the degeneracy factor of the heavy quark (parton).

Due to the different abundances of particle species in a medium at finite chemical potential, it is interesting to study the variation of the heavy-quark interaction rates with the quarks/antiquarks and gluons independently. Figs. 10-(a), (b) and (c) illustrate the dependence of the heavy-quark collisional rates with quarks, antiquarks and gluons of a medium at finite temperature $T$ and quark chemical potential $\mu_{q}$ for an intermediate heavy-quark momentum $(p=5 \mathrm{GeV} / \mathrm{c})$.
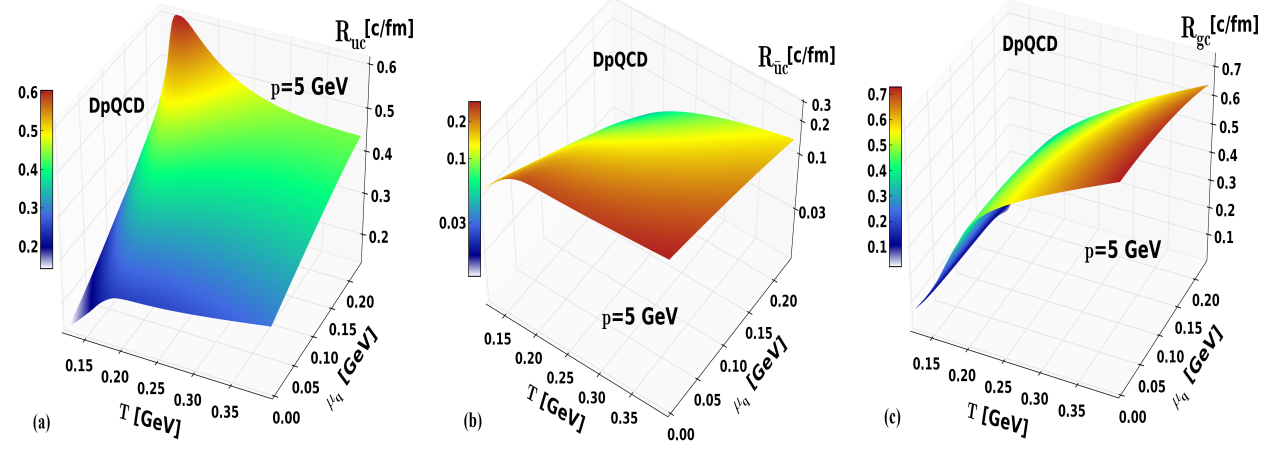

Fig. 10. (Color online) The total elastic interaction rate $R$ of c-quarks in the plasma rest frame as a function of the temperature $T$ and quark chemical potential $\mu_{q}$ due to the scattering with light quarks (a), light antiquarks (b) and gluons (c). The on-shell heavy-quark momentum in all cases is $p=5 \mathrm{GeV} / \mathrm{c}$. The figures are taken from Ref!28

For the case of gluons and antiquarks, the interaction rates are increasing with higher temperature for all $\mu_{q}$. The charm quark interaction rate with light quarks $\left(R_{u c}\right)$ depends on $\left(T, \mu_{q}\right)$ as described in Fig. 10 (a). For larger values of $\mu_{q}$ and small temperatures $\left(T<T_{c}\left(\mu_{q}=0\right)\right), R_{u c}$ is much larger than $R_{\bar{u} c}$ and $R_{g c}$, such 
that the total interaction rates are dominated by $R_{u c}$. On the other hand the $R$ profile is dominated by $R_{g c}$ for small $\mu_{q}$ and large temperatures. This is easy to interpret: At large $\mu_{q}$ the number of light quarks is large compared to the number of antiquarks, i.e. the Fermi-Dirac distribution contributes differently to $R$ for $u$ and $\bar{u}$. On the other hand the gluon number decreases with larger $\mu_{q}$ (relative to light quarks) since it is correlated with the subdominant light antiquarks, via the $T$ and $\mu_{q}$ dependencies of the masses.

\subsection{Diffusion coefficient and energy loss of charm quarks in the hot medium}

Having the matrix elements and the cross sections specified we can calculate transport coefficients $\mathcal{X}$ defined by

$$
\begin{aligned}
\frac{d<\mathcal{X}>}{d t}=\sum_{q, g} & \frac{1}{(2 \pi)^{5} 2 E_{Q}} \int \frac{d^{3} q}{2 E_{q}} f(\boldsymbol{q}) \int \frac{d^{3} q^{\prime}}{2 E_{q^{\prime}}} \int \frac{d^{3} p_{Q}^{\prime}}{2 E_{Q}^{\prime}} \\
& \times \delta^{(4)}\left(P_{\text {in }}-P_{\text {fin }}\right) \mathcal{X} \frac{1}{g_{Q} g_{p}}\left|\mathcal{M}_{2,2}\right|^{2},
\end{aligned}
$$

where $p_{Q}^{\prime}\left(E_{Q}^{\prime}\right)$ is the final momentum (energy) of the heavy quark with the initial energy $E_{Q}$. In Eq. (36) $q\left(E_{q}\right)$ and $q^{\prime}\left(E_{q^{\prime}}\right)$ are the initial and final momenta (energies) of the partons and $f(\boldsymbol{q})$ is their thermal distribution whereas $\left|\mathcal{M}_{2,2}\right|^{2}$ is the transition matrix-element squared for $2 \rightarrow 2$ scattering. Furthermore, in 36 $g_{Q}$ is the degeneracy factor of the heavy quark $\left(g_{Q}=6\right)$ and $g_{p}$ is the degeneracy factor, i.e. $g_{p}=16$ for gluons and $g_{p}=6$ for light quarks. We mention that in Eq. (36) we have discarded Pauli blocking or Bose enhancement factors $\left(1 \pm f\left(\boldsymbol{p}^{\prime}\right)\right.$ in the final states since in our case the occupation numbers $f\left(\boldsymbol{p}^{\prime}\right)$ are rather small in the temperature range of interest due to the rather massive degrees of freedom with pole masses larger than twice the temperature. The errors introduced in this way are smaller than the systematic errors incorporated in $\left|\mathcal{M}_{2,2}\right|^{2}$, i.e. in the transition matrix-element squared. Employing $\mathcal{X}=\left(E-E^{\prime}\right)$ we can calculate the energy loss, $d<E>/ d t\left(p_{Q}, T\right)$, whereas $\mathcal{X}=\left(\boldsymbol{p}_{Q}-\boldsymbol{p}_{Q}^{\prime}\right)$ gives the drag coefficient, $d<\boldsymbol{p}_{Q}>$ $/ d t=A\left(p_{Q}, T\right)$.

The spatial diffusion coefficient $D_{s}$ can be expressed in two different ways ${ }^{69}$ It can be obtained from the slope of the drag coefficient divided by the heavy quark momentum $\eta_{D}=A / p_{Q}$,

$$
D_{s}=\lim _{p_{Q} \rightarrow 0} T /\left(M_{Q} \eta_{D}\right)
$$

as in Ref $\left[68\right.$ It can also be obtained from the diffusion coefficient $\kappa=\frac{1}{3} d<\left(\boldsymbol{p}_{Q}-\right.$ $\left.\boldsymbol{p}_{Q}^{\prime}\right)^{2}>/ d t$, calculated by Eq. $[36)$, as in Ref. ${ }^{70}$

$$
D_{s}=\lim _{p_{Q} \rightarrow 0} \frac{\kappa}{2 M_{Q}^{2} \eta_{D}^{2}} .
$$

Both definitions agree if the Einstein relation is valid. Since in the case of the DpQCD model the deviation from the Einstein relation for small momenta $p_{Q}$ is of 
the order $10-15 \%$ we will adopt eq. $(38)$ for the calculations shown here. We note that the relation (37) is strictly valid in the non-relativistic limit where bremsstrahlung is negligible, i.e. for velocities $\gamma v<1 / \sqrt{\alpha_{s}}$ to leading logarithm in $T / m_{D}$, where $m_{D}$ is the Debye mass. Therefore, it is a good approximation for the interaction of thermal heavy quarks, $M_{Q} \gg T$, with a typical thermal momentum $p \sim \sqrt{M T}$ and a velocity $v \sim \sqrt{T / M} \ll 1$.
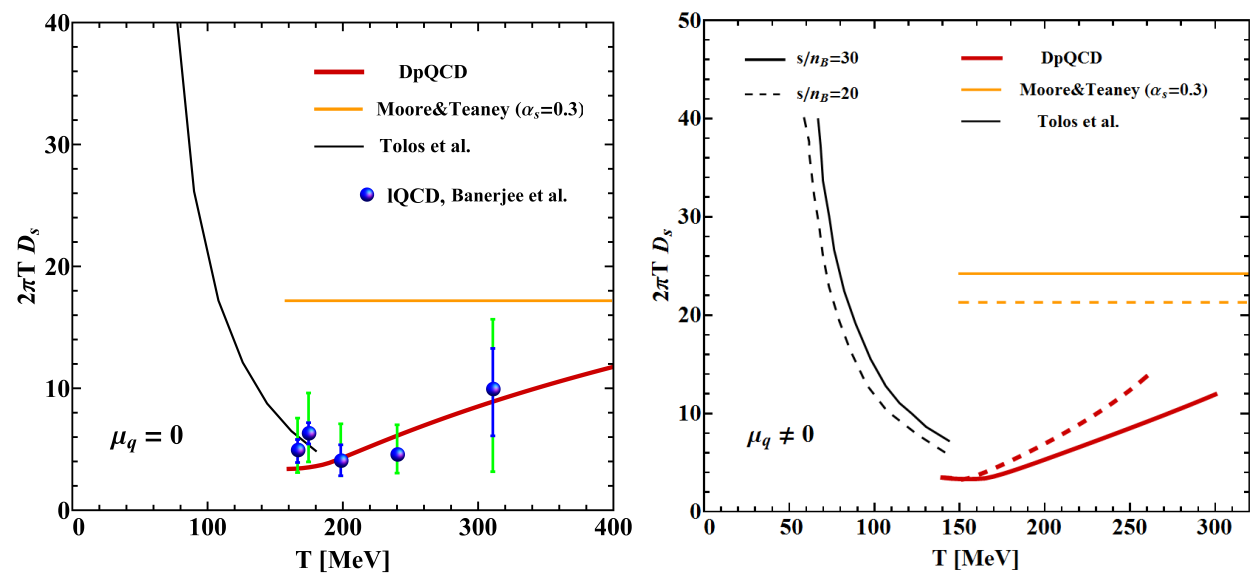

Fig. 11. (Color online) (l.h.s.) Spatial diffusion coefficient for heavy quarks, $D_{s}$, as a function of $T$ for $\mu_{q}=0$. Below $T=180 \mathrm{MeV}$ we display the hadronic diffusion coefficient from Ref.70 above $T=180 \mathrm{MeV}$ that for a partonic environment. The solid orange line is the result of Ref while the red thick solid line shows the DpQCD prediction. The lattice calculations are from Ref ${ }^{71}$ (r.h.s.) Spatial diffusion constant, $D_{s}$, as a function of $T$ for $\mu_{q} \neq 0 . D_{s}$ is displayed for different values of $s / n_{B}$ for a hadronic environment ${ }^{70}$ as well as for a partonic environment. For the latter pQCD calculations are confronted with DpQCD calculations. The figures are taken from Ref ${ }^{[72}$

In Fig. 11 (l.h.s.) we display the spatial diffusion coefficient $D_{s}(38)$ as a function of $T$ for $\mu_{q}=0$. Our results are compared with the leading order (LO) results obtained by Moore and Teaney ${ }^{69}$ for perturbative partons and $\alpha_{s}=0.3$ as well as with the lattice calculations from Ref. ${ }^{71}$ for temperatures above $T_{c}$. We mention that a Debye mass is included in the Moore and Teaney calculation ${ }^{69}$ for the scattering of heavy quarks on the QGP partons and leads to a finite cross section at vanishing 4-momentum transfer. This Debye mass is generated dynamically and in some sense can be considered as the analogue to the DQPM pole masses. The lattice results in Fig. 11 (a) have recently been confirmed by the Bielefeld collaboration.

The spatial diffusion coefficient in deconfined matter is compared with the result for the spatial diffusion coefficient of a heavy meson in hadronic matter ${ }^{70}$ in Fig 11 (l.h.s.) for temperatures below $T_{c}$. We observe that at $T \approx T_{c}$ the spatial diffusion coefficients for hadronic and partonic matter join almost continuously and agree with the lattice results. On the other hand, pQCD calculations yield a larger value 
of the spatial diffusion coefficient as compared to the DpQCD model leading to a discontinuity of $D_{s}$ close to $T_{c}$. Rapp et al ${ }^{73 \mid 74}$ have shown that the spatial diffusion coefficient in pQCD calculations can be lowered by adding nonperturbative heavy-quark interactions. Also hard thermal loop calculations with 'effective' Debye masses and a running coupling lead to a substantial lowering of $D_{s}$ and bring its values to the vicinity of the lattice results. $[68$

The DpQCD calculations can be extended to finite $\mu_{q}$ assuming adiabatic trajectories (constant entropy per net baryon $s / n_{B}$ ) for the expansion. The latter is calculated using the pole masses of the plasma constituents in the DQPM model as well as for perturbative partons. For a given $s / n_{B}$ the chemical potential $\mu_{B}$ is a monotonic function of $T$ and therefore we can display $D_{s}$ as a function of $T$ and $s / n_{B}$. Fig. 11 (r.h.s.) displays the spatial diffusion coefficient for finite chemical potential, i.e for different values of the entropy per net baryon $s / n_{B}$. The pQCD calculations are obtained by adding the chemical potential to the thermal distributions and to the Debye mass when calculating the pQCD drag and diffusion coefficients (cf. Eq. (B13) of Ref.69). We observe - as in the $\mu_{q}=0$ case - that the DpQCD spatial diffusion coefficient of heavy quarks approximately joins smoothly those of the hadron gas. (We expect that in the $\mu_{B}$ region investigated here the transition remains a cross over transition). On the contrary, pQCD calculations close to $T_{c}$ are a factor of 3 higher leading to a discontinuity of the spatial diffusion coefficient, which is not compatible with a cross-over transition as predicted by lattice calculations. This is a strong indication that close to the phase transition the effective degrees-of-freedom should be massive quasi-particles and not perturbative quarks and gluons.

For comparing our model predictions with experimental data another transport coefficient, the energy loss of a heavy quark per unit length, $d<E>/ d x=$ $d\langle E\rangle / v d t$, is important. It can be obtained from Eq. (36) by the choice $\mathcal{X}$ $=\left(E_{Q}-E_{Q}^{\prime}\right)$. The energy loss of a heavy quark with an incoming momentum of $10 \mathrm{GeV} / \mathrm{c}$ as a function of $T$ and $\mu_{q}$ in the DpQCD approach is presented in Fig. 12 As expected for a cross-over transition we observe a very smooth dependence on both variables, $T$ and $\mu_{q}$. For $\mu_{q}=0$ the gluon pole mass depends on the temperature and therefore the increase of the energy loss is due to the change of the running coupling $g^{2}\left(T / T_{c}\right)$. For $\mu_{q}=0.2 \mathrm{GeV}$, the energy loss is also increasing with temperature but less than for $\mu_{q}=0$ because here the increase of the coupling is partially counterbalanced by the decrease of the gluon pole mass.

We recall that the properties of the QCD medium in terms of the shear viscosity over entropy ratio $\eta / s$ as well as the electric conductivity over temperature $\sigma_{e} / T$ show a minimum close to $T_{c}$ (cf. Section 4) which apparently repeats in the charm spatial diffusion coefficient reflecting a maximum in the interaction strength $g^{2}\left(T / T_{c}\right)$ of the QCD degrees of freedom at temperatures close to $T_{c}$. 


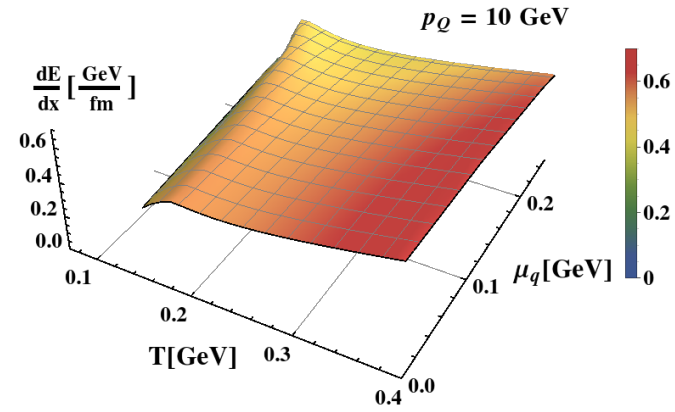

\section{Summary}

We have presented in this review an extended dynamical quasiparticle model $\left(\mathrm{DQPM}^{*}\right)$ incorporating momentum-dependent selfenergies in the parton propagators which are reflected in momentum-dependent masses and widths. Accordingly, the QGP effective degrees of freedom appear as interacting off-shell quasi-particles with masses and widths that depend on three-momentum $\boldsymbol{p}$, temperature $T$ and chemical potential $\mu_{q}$ as given in Eqs. (3). These expressions provide a proper high temperature limit (as in the HTL approximation) and approach the pQCD limit for large momenta $|\boldsymbol{p}|$. As in the standard DQPM the effective coupling is enhanced in the region close to $T_{c}$, which leads to an increase of the parton masses roughly below $1.2 T_{c}$ (cf. Fig. 1 (a)).

The extended dynamical quasiparticle model DQPM* reproduces quite well the lQCD results, i.e. the QGP equation of state, the baryon density $n_{B}$ and the quark susceptibility $\chi_{q}$ at finite temperature $T$ and quark chemical potential $\mu_{q}$ which had been a challenge for quasiparticle models so far ${ }^{14}$ (see also Fig. $4 \mathrm{~b}$ ). A detailed comparison between the available lattice data and DQPM* results indicates a very good agreement for temperatures above $\sim 1.2 T_{c}$ in the pure partonic phase and therefore validates our description of the QGP thermodynamic properties. For temperatures in the vicinity of $T_{c}$ (and $\mu_{B}=400 \mathrm{MeV}$ ) we cannot expect our model to work so well since here hadronic degrees of freedom, which are discarded in the $\mathrm{DQPM}^{*}$, mix in a crossover phase.

Furthermore, we have computed also the QGP shear viscosity $\eta$, the bulk viscosity $\zeta$, and the electric conductivity $\sigma_{e}$ at finite temperature and chemical potential in order to probe some transport properties of the medium. The relaxation times at finite temperature and chemical potential, used in our study, are evaluated for the dynamical quasi-particles using the parton width which is averaged over the thermal ensemble at fixed $T$ and $\mu_{q}$. We, furthermore, emphasize the importance of nonperturbative effects near $T_{c}$ to achieve a small $\eta / s$ as supported by different phenomenological studies and indirect experimental observations as well as a maximum in the ratio $\zeta / s$. When comparing our results for $\eta / s$ to those from the standard DQPM (with momentum-independent selfenergies) in Ref.25] we find a close agree- 
ment. In the $\mathrm{DQPM}^{*}$ the gluon mass is slightly higher (for low momenta) and the quark mass is slightly smaller than in the DQPM. Furthermore, the interaction widths are somewhat larger in the DQPM* which finally leads to a slightly lower shear viscosity $\eta$ than in the DQPM. This also holds for the electric conductivity $\sigma_{e}$ which in the DQPM* gives results even closer to the present lQCD 'data'.

Additionally, we have reported on the results of the momentum-independent DQPM for the description of heavy quarks $Q$ in the hot partonic medium and studied their transport properties, i.e. the spatial diffusion coefficient $D_{s}$ and the energy loss $d E / d x$ also at finite $T$ and $\mu_{q}$. A medium at finite chemical potential leads to a reduction of the $q Q$ and $g Q$ elastic cross section and consequently to a reduction of heavy-quark energy and momentum losses as compared to a medium at $\mu_{q}=0$. Nevertheless, we have concluded that longitudinal momentum transfers are important not only in a hot medium but also in a dense medium whereas the dense medium leads to less transverse fluctuations in the heavy quark propagation. The relative large drag at low temperatures is due to the strong increase of the running coupling $\alpha_{s}\left(T, \mu_{q}\right)$ (infrared enhancement) for temperatures close to $T_{c}\left(\mu_{q}\right)$.

Furthermore, we have observed a smooth dependence of the energy loss $d E / d x$ on both variables $T$ and $\mu_{q}$ at finite but not too large values of $\mu_{q}$. Such a profile is expected for a cross-over transition from the partonic to the hadronic medium. For $\mu_{q}=0$ the gluon mass depends on the temperature and therefore the increase of the energy loss is due to a change of the coupling. For $\mu_{q}=0.2 \mathrm{GeV}$, the energy loss is also increasing with temperature but less than for $\mu_{q}=0$ because here both the coupling and the effective gluon mass decrease and the increase of the infrared regulator is counterbalanced by the decrease of the coupling. Since the variations of all transport coefficients with $T$ and $\mu_{q}$ are rather smooth (within the present DQPM/DQPM* propagators) the transition from hadronic degrees of freedom to partonic ones remains a crossover up to $\mu_{q}=0.2 \mathrm{GeV}$.

In view of our results on the description of the QGP thermodynamics and transport properties, one can conclude that the $\mathrm{DQPM}^{*}$ provides a promising approach to study the QGP in equilibrium at finite temperature $T$ and chemical potential $\mu_{q}$. Moreover, we have demonstrated that one can simultaneously reproduce the lQCD pressure, the quark susceptibility and the QCD transport properties using a dynamical quasi-particle picture for the QGP effective degrees of freedom that allows for a transparent interpretation of the various results from lattice QCD.

We recall that a covariant transport approach has been set up a couple of years ago in Refs 33 in which the description of the partonic phase has been based on the partonic propagators of the standard DQPM. This approach is denoted by partonhadron-string-dynamics (PHSD) and has been employed for the description of $p+p$, $p+A$ and $A+A$ reactions at invariant energies from $\sqrt{s_{N N}} \approx 8 \mathrm{GeV}$ to $5 \mathrm{TeV}$. For a recent review on bulk and electromagnetic probes we refer the reader to Ref. ${ }^{75}$ and for an application to the charm sector at RHIC and LHC energies to Refs. 76$] 77$ Since the DQPM* provides appropriate propagators also for finite quark chemical 
potentials $\mu_{q}$, a related implementation in the PHSD is foreseen and will allow to investigate the phase boundary in heavy-ion collisions also at lower bombarding energies (FAIR/NICA) where baryonic effects and chiral symmetry restoration in the hadronic phase are expected to dominate ${ }^{78}$

\section{Acknowledgements}

The authors acknowledge valuable discussions with R. Marty, P. B. Gossiaux, J. Aichelin, O. Linnyk, P. Moreau, A. Palmese, E. Seifert and T. Song. This work has been supported by the "HIC for FAIR" framework of the "LOEWE" program. The computational resources have been provided by the LOEWE-CSC.

\section{References}

1. S. Borsanyi, Z. Fodor, C. Hoelbling, S. D. Katz, S. Krieg, C. Ratti and K. K. Szabo, PoS LATTICE2014 (2015) 224.

2. S. Borsanyi, Z. Fodor, C. Hoelbling, S. D. Katz, S. Krieg et al., Phys. Lett. B730 (2014) 99.

3. S. Borsanyi, Z. Fodor, C. Hoelbling, S. D. Katz, S. Krieg and K. K. Szabo, PoS LATTICE2013 (2014) 155.

4. Wuppertal-Budapest Collaboration (S. Borsanyi et al.), JHEP 1009 (2010) 073.

5. S. Borsanyi, G. Endrodi, Z. Fodor, A. Jakovac, S. D. Katz et al., JHEP 1011 (2010) 077.

6. R. A. Soltz, C. DeTar, F. Karsch, S. Mukherjee and P. Vranas, Ann. Rev. Nucl. Part. Sci. 65 (2015) 379.

7. M. I. Gorenstein and S.-N. Yang, Phys. Rev. D52 (1995) 5206.

8. P. Levai and U. W. Heinz, Phys. Rev. C57 (1998) 1879.

9. A. Peshier, B. Kämpfer and G. Soff, Phys. Rev. C61 (2000) 045203.

10. A. Peshier, B. Kämpfer and G. Soff, Phys. Rev. D66 (2002) 094003.

11. V. M. Bannur, Eur. Phys. J. C50 (2007) 629.

12. P. K. Srivastava, S. K. Tiwari and C. P. Singh, Phys. Rev. D82 (2010) 014023.

13. M. Bluhm, B. Kämpfer and G. Soff, Phys. Lett. B620 (2005) 131.

14. S. Plumari, W. M. Alberico, V. Greco and C. Ratti, Phys. Rev. D84 (2011) 094004.

15. A. Peshier and W. Cassing, Phys. Rev. Lett. 94 (2005) 172301.

16. W. Cassing, Nucl. Phys. A 791 (2007) 365.

17. W. Cassing, Nucl. Phys. A795 (2007) 70.

18. W. Cassing, Eur. Phys. J. ST 168 (2009) 3.

19. R. Marty, E. Bratkovskaya, W. Cassing, J. Aichelin and H. Berrehrah, Phys. Rev. C88 (2013) 045204.

20. S. Borsanyi, G. Endrodi, Z. Fodor, S. Katz, S. Krieg et al., JHEP 1208 (2012) 053.

21. G. Endrodi, Z. Fodor, S. D. Katz and K. K. Szabo, JHEP 04 (2011) 001.

22. A. Bazavov, T. Bhattacharya, M. Cheng, N. Christ, C. DeTar et al., Phys. Rev. D80 (2009) 014504.

23. ALICE Collaboration (B. Abelev et al.), JHEP 09 (2012) 112.

24. ALICE Collaboration (B. Abelev et al.), Phys. Rev. Lett. 111 (2013) 102301.

25. V. Ozvenchuk, O. Linnyk, M. Gorenstein, E. Bratkovskaya and W. Cassing, Phys. Rev. C87 (2013) 064903.

26. H. Berrehrah, E. Bratkovskaya, W. Cassing, P. Gossiaux, J. Aichelin et al., Phys. Rev. C89 (2014) 054901. 
27. H. Berrehrah, E. Bratkovskaya, W. Cassing and R. Marty, J. Phys. Conf. Ser. 612 (2015) 012050.

28. H. Berrehrah, E. Bratkovskaya, W. Cassing, P. Gossiaux and J. Aichelin, Phys. Rev. C91 (2015) 054902.

29. G. Aarts and J. M. Martinez Resco, JHEP 02 (2004) 061.

30. G. Aarts and J. M. Martinez Resco, JHEP 04 (2002) 053.

31. W. Cassing and E. Bratkovskaya, Phys. Rev. C78 (2008) 034919.

32. B. Vanderheyden and G. Baym, J. Stat. Phys. 93 (1998) 843.

33. E. Bratkovskaya, W. Cassing, V. Konchakovski and O. Linnyk, Nucl. Phys. A 856 (2011) 162

34. A. Peshier, J. Phys. G31 (2005) S371.

35. C. S. Fischer, J. Phys. G32 (2006) R253.

36. C. Bonati, M. D'Elia, M. Mariti, M. Mesiti, F. Negro et al., Phys. Rev. D90 (2014) 114025 .

37. L. Rauber and W. Cassing, Phys. Rev. D89 (2014) 065008.

38. W. Cassing and E. Bratkovskaya, Nucl. Phys. A831 (2009) 215.

39. H. Berrehrah, W. Cassing, E. Bratkovskaya and T. Steinert, Phys. Rev. C93 (2016) 044914.

40. J. P. Blaizot, E. Iancu and A. Rebhan, Phys. Rev. D63 (2001) 065003.

41. C. Allton, S. Ejiri, S. Hands, O. Kaczmarek, F. Karsch et al., Phys. Rev. D68 (2003) 014507.

42. C. Allton, M. Doring, S. Ejiri, S. Hands, O. Kaczmarek et al., Phys. Rev. D71 (2005) 054508.

43. P. Chakraborty and J. Kapusta, Phys. Rev. C83 (2011) 014906.

44. S. Plumari, A. Puglisi, F. Scardina and V. Greco, Phys. Rev. C86 (2012) 054902.

45. N. Christiansen, M. Haas, J. M. Pawlowski and N. Strodthoff, Phys. Rev. Lett. 115 (2015) 112002.

46. L. P. Csernai, J. Kapusta and L. D. McLerran, Phys. Rev. Lett. 97 (2006) 152303.

47. G. Policastro, D. T. Son and A. O. Starinets, Phys. Rev. Lett. 87 (2001) 081601.

48. P. Kovtun, D. T. Son and A. O. Starinets, Phys. Rev. Lett. 94 (2005) 111601.

49. H. B. Meyer, Phys. Rev. D76 (2007) 101701.

50. A. Nakamura and S. Sakai, Phys. Rev. Lett. 94 (2005) 072305.

51. S. Sakai and A. Nakamura, PoS LAT2007 (2007) 221.

52. W. Cassing, O. Linnyk, T. Steinert and V. Ozvenchuk, Phys. Rev. Lett. 110 (2013) 182301.

53. T. Steinert and W. Cassing, Phys. Rev. C89 (2014) 035203.

54. O. Linnyk, V. Konchakovski, T. Steinert, W. Cassing and E. L. Bratkovskaya, Phys. Rev. C92 (2015) 054914.

55. H.-T. Ding, A. Francis, O. Kaczmarek, F. Karsch, E. Laermann et al., Phys. Rev. D83 (2011) 034504.

56. G. Aarts, C. Allton, J. Foley, S. Hands and S. Kim, Phys. Rev. Lett. 99 (2007) 022002.

57. S. Gupta, Phys. Lett. B597 (2004) 57.

58. O. Kaczmarek and M. Müller, PoS LATTICE2013 (2014) 175.

59. B. B. Brandt, A. Francis, H. B. Meyer and H. Wittig, PoS ConfinementX (2012) 186.

60. P. Buividovich, M. Chernodub, D. Kharzeev, T. Kalaydzhyan, E. Luschevskaya et al., Phys. Rev. Lett. 105 (2010) 132001.

61. G. Aarts, C. Allton, A. Amato, P. Giudice, S. Hands et al., JHEP 1502 (2015) 186.

62. S.-x. Qin, Phys. Lett. B742 (2015) 358.

63. P. B. Arnold, G. D. Moore and L. G. Yaffe, JHEP 0305 (2003) 051. 
64. H. D. Politzer, Phys. Rep. 14 (1974) 129.

65. R. Cutler and D. Sivers, Phys. Rev. D 17 (1978) 196.

66. B. Combridge, Nucl. Phys. B 151 (1979) 429 .

67. B. Combridge, J. Kripfganz and J. Ranft, Phys. Lett. B 70 (1977) 234.

68. H. Berrehrah, P.-B. Gossiaux, J. Aichelin, W. Cassing and E. Bratkovskaya, Phys. Rev. C90 (2014) 064906.

69. G. D. Moore and D. Teaney, Phys. Rev. C71 (2005) 064904.

70. L. Tolos and J. M. Torres-Rincon, Phys. Rev. D88 (2013) 074019.

71. D. Banerjee, S. Datta, R. Gavai and P. Majumdar, Phys. Rev. D85 (2012) 014510.

72. H. Berrehrah, P. Gossiaux, J. Aichelin, W. Cassing, J. M. Torres-Rincon and E. Bratkovskaya, Phys. Rev. C90 (2014) 051901.

73. R. Rapp and H. van Hees, Heavy Quarks in the Quark-Gluon Plasma, in R. C. Hwa, X.-N. Wang (Ed.) Quark Gluon Plasma 4, World Scientific, 111 , (2010).

74. M. He, R. J. Fries and R. Rapp, Phys. Rev. Lett. 110 (2013) 112301.

75. O. Linnyk, E. L. Bratkovskaya and W. Cassing, Prog. Part. Nucl. Phys. 87 (2016) 50 .

76. T. Song, H. Berrehrah, D. Cabrera, W. Cassing and E. Bratkovskaya, Phys. Rev. C93 (2016) 034906.

77. T. Song, H. Berrehrah, D. Cabrera, J. M. Torres-Rincon, L. Tolos, W. Cassing and E. Bratkovskaya, Phys. Rev. C92 (2015) 014910.

78. W. Cassing, A. Palmese, P. Moreau and E. L. Bratkovskaya, Phys. Rev. C93 (2016) 014902 . 